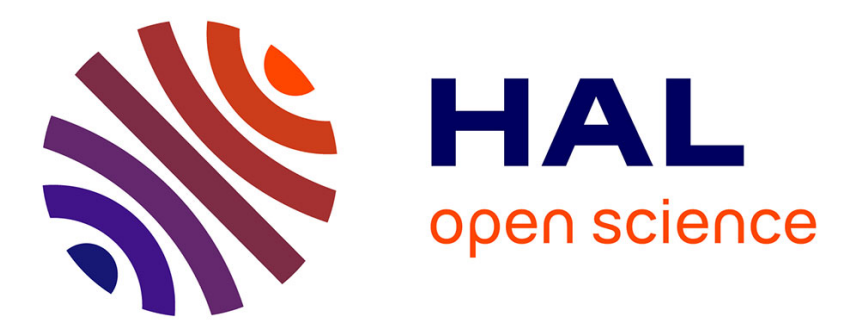

\title{
Consumption minimization for an academic model of a vehicle
}

Ouazna Oukacha, Nicolas Boizot

\section{To cite this version:}

Ouazna Oukacha, Nicolas Boizot. Consumption minimization for an academic model of a vehicle. Optimal Control Applications and Methods, 2020, 41 (4), pp.1288-1320. 10.1002/oca.2604 . hal$01384651 \mathrm{v} 3$

\section{HAL Id: hal-01384651 \\ https://hal.science/hal-01384651v3}

Submitted on 28 May 2020

HAL is a multi-disciplinary open access archive for the deposit and dissemination of scientific research documents, whether they are published or not. The documents may come from teaching and research institutions in France or abroad, or from public or private research centers.
L'archive ouverte pluridisciplinaire HAL, est destinée au dépôt et à la diffusion de documents scientifiques de niveau recherche, publiés ou non, émanant des établissements d'enseignement et de recherche français ou étrangers, des laboratoires publics ou privés. 


\title{
Consumption minimisation for an academic model of a vehicle
}

\author{
Ouazna Oukacha*and Nicolas Boizot ${ }^{\dagger}$
}

Supported by the French National Research Agency, ANR (SRGI project)ANR-15-CE40-0018.

\begin{abstract}
The present article is a study of an optimal control problem having a non-differentiable, but Lipschitz, cost function. It is inspired by the minimisation of the energy consumption of a car-like vehicle or robot along a road which profile is known. This problem is stated by means of a simple model of the longitudinal dynamics and a running cost that comprises both an absolute value function and a function that accounts for the efficiency of the energy conversion process. A regularity result that excludes chattering phenomena from the set of solutions is proven. It is valid for the class of control affine systems, which includes the considered problem. Three case studies are detailed and analysed. The optimal trajectories are shown to be made of bang-bang, inactivated, singular and backward arcs.
\end{abstract}

energy consumption minimisation; singular arcs; regularity; non-differentiable cost function.

\section{Introduction}

This article presents a study of an optimal control problem having a non-differentiable cost for certain values of the control or of the state. One of the most notable class of such problems is known as $\mathrm{L}^{1}$-minimisation and consists of the minimisation of the $\mathrm{L}^{1}$-norm of the control:

$$
\int_{0}^{T_{f}}|u(t)| d t \rightarrow \min
$$

As it is well-known, cf. [2, 5, 8, 9, 16, 18, 20], such a cost structure implies inactivations, that is, non-trivial intervals of time where the control vanishes. As mentioned in [9, 20], this behaviour seems relevant when considering energy consumption minimisation in the sense that inactivations act as a selection process that singles out moments when the control variable is the most efficient.

The problem addressed in this paper is an "academic" model of the optimal energy consumption of a car-like vehicle or robot along a road for which the profile is known. It follows the ideas of [4] regarding the minimisation of the absolute work of an actuated mechanical system. For instance, if $x(t)$ is a displacement and $u(t)$ an external force, the absolute work of $u$ is just

$$
\int_{0}^{T_{f}}|\dot{x}(t) u(t)| d t
$$

In the paper, the running cost is derived from this latter expression with the help of a yield function that translates the efficiency of the energy conversion process. As it is discussed and

\footnotetext{
*Université Polytechnique Hauts-de-France, LAMIH, CNRS UMR 8201, LAMIH, Valenciennes, France.

†Laboratoire d'Informatique et des Systèmes, Université de Toulon, Aix Marseille Univ, CNRS UMR 7020, LIS, Marseille, France. E-mail: boizot@univ-tln.fr
} 
highlighted in the rest of the article, optimal strategies are made of classic bang-bang controls and inactivations, but may also include singular arcs that cannot be obtained from the successive time derivatives of the switching function (e.g. [3, 11]).

In Section 2, the problem under consideration is stated through the derivation of both the model of the vehicle dynamics and the running cost. Section 3 is dedicated to two regularity issues, namely, a theorem that allows us to exclude chattering phenomena and a lemma that allows us to restrict the set of candidate trajectories for optimality. Theorem 1, which purpose is to allow us to handle the chattering problem is, in some sense, complementary to recent strategies developed in the framework of direct methods for optimal control. In particular, interested reader may refer to $[?, ?]$, and references herein, where a penalty term that measures the variations of the control variable (called the total variation) is added to the cost. This strategy results in an optimal control problem which is non-smooth with respect to the control variable and dealt with using control parameterization techniques.

Finally, three case studies are detailed in Section 4. The first case, for a flat road and without friction, is treated in Section 4.1. It is very simple since optimal trajectories are always obtained from bang-bang controls and inactivations only. Although counterintuitive, in this case, the optimal strategy doesn't depend on the yield function used. In the second case study, where frictions are now considered, optimal trajectories having singular arcs are observed. The presence of such arcs depends more on the value of the final time than on the yield function itself. Still, this latter has a noticeable influence on the result, as it is highlighted in Section 4.2. The third case study is addressed in Section 4.3, where a non-flat road profile and no friction coefficient are considered. On top of all the previous phenomena, we display optimal trajectories that require the vehicle to travel backward for some time.

\section{Problem statement}

In the following, in order to simplify the notations, the time dependency of time varying functions is omitted as soon as there is no ambiguity. The sign function is denoted by sgn, and $X^{t}$ is the transpose of vector $X$.

\subsection{Vehicle dynamics}

The vehicle travels on a road represented in a position-height plane as it is sketched in Figure 1 (see e.g. [12] for general considerations regarding vehicle dynamics, and [?, 19] for models that take into account both the longitudinal and the lateral dynamics). Its coordinates are denoted by $(\xi, h)$ and $\theta$ is the orientation. We assume that only the four following forces act on the vehicle and that the tyre slip is negligible:

- the gravity $\mathbf{G}$, whose norm equals the gravitational constant $g$ times the mass of the car $m$;

- the reaction of the road $\mathbf{R}$, whose norm equals $m g \cos (\theta)$;

- the propulsion force $\mathbf{F}$, whose norm is the control variable $u(t)$;

- the drag force $\mathbf{f}$ opposes motion and, in this work, we consider fluid friction only with a norm proportional to speed: $\|\mathbf{f}\|=\alpha \sqrt{\dot{\xi}^{2}+\dot{h}^{2}}$ where $\alpha \geq 0$ is the drag coefficient. 
The dynamics are given by Newton's second law of motion:

$$
m\left(\begin{array}{c}
\ddot{\xi} \\
\ddot{h}
\end{array}\right)=\left(\begin{array}{c}
u \cos (\theta) \\
u \sin (\theta)
\end{array}\right)-\left(\begin{array}{c}
0 \\
m g
\end{array}\right)-\left(\begin{array}{c}
\alpha \operatorname{sgn}(\dot{\xi}) \sqrt{\dot{\xi}^{2}+\dot{h}^{2}} \cos (\theta) \\
\alpha \operatorname{sgn}(\dot{\xi}) \sqrt{\dot{\xi}^{2}+\dot{h}^{2}} \sin (\theta)
\end{array}\right)+\left(\begin{array}{c}
-m g \cos (\theta) \sin (\theta) \\
m g \cos ^{2}(\theta)
\end{array}\right)
$$
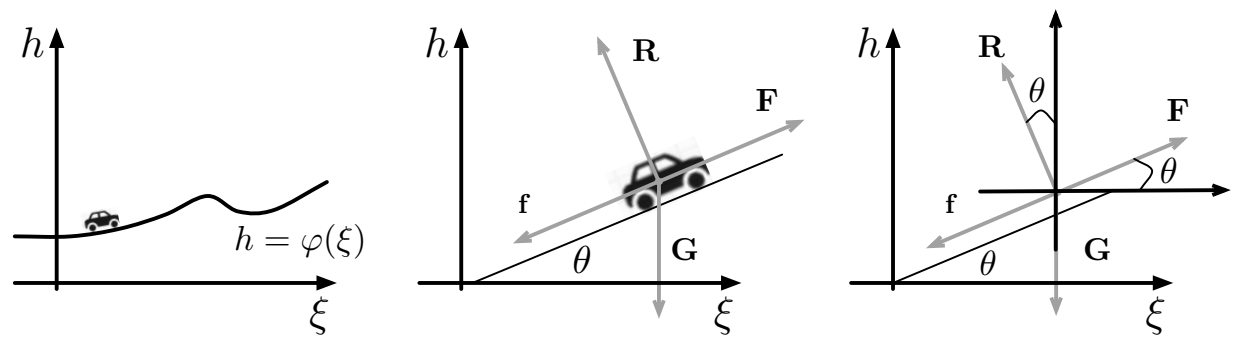

Figure 1: Modelling and notations.

We assume that the road profile is given by a $\mathcal{C}^{1}$ function $h=\varphi(\xi)$. Since the angle $\theta$ belongs to the interval ] $-\frac{\pi}{2}, \frac{\pi}{2}\left[\right.$, it is uniquely determined by $\theta(\xi)=\arctan \left(\varphi^{\prime}(\xi)\right)$ where $\varphi^{\prime}$ denotes the derivative with respect to $\xi$. Thus, system (1) has in fact only one degree of freedom whose dynamics are:

$$
\left\{\begin{aligned}
m \ddot{\xi} & =u \cos (\theta(\xi))-\alpha \dot{\xi} \sqrt{1+\left(\varphi^{\prime}(\xi)\right)^{2}} \cos (\theta(\xi))-m g \cos (\theta(\xi)) \sin (\theta(\xi)) \\
\theta(\xi) & =\arctan \left(\varphi^{\prime}(\xi)\right)
\end{aligned}\right.
$$

Let $x$ be the curvilinear abscissa of $(\xi, h(\xi))$ and $y$ be equal to $\dot{x}$-i.e. $x=\int_{0}^{\xi} \sqrt{1+\left(\varphi^{\prime}(z)\right)^{2}} d z$ and $y=\dot{\xi} \sqrt{1+\left(\varphi^{\prime}(\xi)\right)^{2}}$. Then, system (2) is rewritten:

$$
\left\{\begin{aligned}
\dot{x} & =y \\
m \dot{y} & =u-\alpha y-m g \sin (\theta(x))
\end{aligned}\right.
$$

where both $x$ and $y$ are absolutely continuous functions on $\mathbb{R}, u(t) \in L^{\infty}([0, T], \mathbb{R})$ and $\theta(x)$ is the $\mathcal{C}^{1}$ function that accounts for the road profile $-h=\varphi(\xi)$ is recovered from $\theta(x)[?]$.

\subsection{Cost function}

The cost function is the energy consumed to steer the vehicle from a starting position at time 0 to some final position at time $T_{f}$. Let $D(t)$ be the energy flow rate, then the cost function is simply

$$
\int_{0}^{T_{f}} D(\tau) d \tau
$$

Now, let $A$ be the absolute work of the propulsion force. It is given by [4]

$$
A=\int_{0}^{T_{f}}|\dot{\xi} u| \sqrt{1+\left(\varphi^{\prime}(\xi)\right)^{2}} d \tau
$$

The associated absolute instantaneous power is $\frac{d A}{d t}$ and is connected to the flow rate with the help of a yield function $\rho($.$) : \frac{d A}{d t}=D(t) \rho($.$) . We assume that the yield function is as follows,$ with examples displayed in Figure 2: 
- its argument is the speed of the vehicle -i.e. $y=\dot{\xi} \sqrt{1+\left(\varphi^{\prime}(\xi)\right)^{2}}$;

- it takes its values in the interval $] 0,1]$, for all $y \in \mathbb{R}$;

- it has one of the two following behaviours for all $y \in \mathbb{R}^{+}$(respectively $\mathbb{R}^{-}$):

1. $\rho(y)$ is identically constant;

2. $\rho(y)$ admits a maximum at $y^{+}$, is increasing on $] 0, y^{+}[$and decreasing on $] y^{+},+\infty[$; $\left(\rho(y)\right.$ admits a maximum at $y^{-}$, is decreasing on $] y^{-}, 0[$ and increasing on $]-\infty, y^{-}[)$;

- it is differentiable as many times as needed.

Finally, the cost function is denoted by $J(u)$ and has the following expression:

$$
J(u)=\int_{0}^{T_{f}} \frac{|\dot{\xi} u|}{\left.\rho\left(\dot{\xi} \sqrt{1+\left(\varphi^{\prime}(\xi)\right.}\right)^{2}\right)} \sqrt{1+\left(\varphi^{\prime}(\xi)\right)^{2}} d \tau=\int_{0}^{T_{f}} \frac{|y u|}{\rho(y)} d \tau
$$

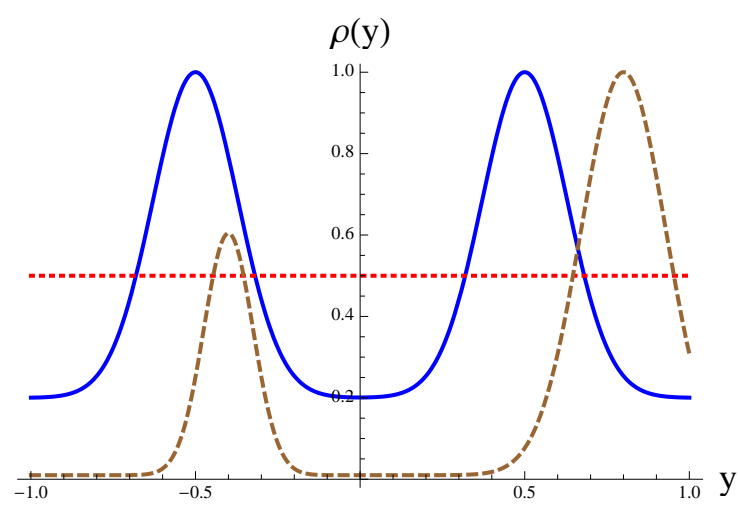

Figure 2: Yield function examples.

Remark 1. The proposed model for the running cost may not feel very realistic since the energy consumption is the same while accelerating $(u>0)$ or braking $(u<0)$. First of all, let us mention that our goal is to capture the influence of the non-differentiability on the optimal strategy. As such, electrical engines equipped with regenerative braking devices are not considered for simplicity. On the other side, if one wants to better model internal combustion engines, where braking costs almost nothing, phases when $u<0$ can be discriminated with the help of a function of the form:

$$
v(u)=\left\{\begin{array}{ll}
\alpha u & \text { for } u \geq 0 \\
-\beta u & \text { for } u<0
\end{array} \quad \text { with } \quad 0<\beta \leq \alpha \quad \text { and } \quad J(u)=\int_{0}^{T_{f}} \frac{|y| v(u)}{\rho(y)} d \tau\right.
$$

This new cost function will have an impact on the several calculations and on the switching times, but not on the qualitative behaviours displayed in the remainder of the article. 


\subsection{A fixed-time optimal control problem}

In the remainder of this paper, we restrict our study to trajectories having a forward destination and a fixed final time $T_{f}$. Also, in order to ease the exposure of the study, we set the parameters $m$ and $g$ to 1 . The optimal control problem under consideration is:

$$
\left(\mathcal{P}_{1}\right) \begin{cases}\text { Minimise } & J(u)=\int_{0}^{T_{f}} \frac{|y u|}{\rho(y)} d \tau \\
\text { Subject to } & \left(\Sigma_{1}\right)\left\{\begin{array}{l}
\dot{x}=y \\
\dot{y}=u-\alpha y-\sin (\theta(x)) \quad \text { for } t \in\left[0, T_{f}\right]
\end{array}\right. \\
& X(0)=\left(\begin{array}{c}
x(0) \\
y(0)
\end{array}\right)=\left(\begin{array}{c}
0 \\
0
\end{array}\right), X\left(T_{f}\right)=\left(\begin{array}{c}
x\left(T_{f}\right) \\
y\left(T_{f}\right)
\end{array}\right)=\left(\begin{array}{c}
x_{f} \\
0
\end{array}\right), \text { with } x_{f}>0 \\
& |u(t)| \leq 1 \text { for } t \in\left[0, T_{f}\right] \\
& T_{f}>0 \text { is fixed. }\end{cases}
$$

Following [15, Thm. 4, P. 259], and also [4, 5], the set of solutions to this problem is non-empty as soon as $T_{f}$ is greater or equal to the minimum time problem associated to $\left(\mathcal{P}_{1}\right)$.

Let us remark that, in order to perform a relevant study in free time, $\left(\mathcal{P}_{1}\right)$ requires a specific road profile, a cost that penalises the travel time or a specific yield function $\rho(y)$. This statement is illustrated in the case of a flat road (i.e. $\theta \equiv 0$ ) by the following proposition.

Proposition 1. Let us consider problem $\left(\mathcal{P}_{1}\right)$ with a flat road (that is to say $\theta \equiv 0$ ). If the final time $T_{f}$ is free then the optimal strategy lies in the set of control variables for which the associated cost is null.

Proof. In order to prove this statement, we show that for any strategy having a finite final time and a strictly positive cost, there exists another strategy (also with a finite final time) having a smaller associated cost. In order to do so, we just construct two one-parameter families of control strategies (with and without friction) such that the associated cost goes to zero when the parameter tends toward zero.

We also show that the final time associated to those strategies goes to infinity when the parameter vanishes. Therefore, the optimal solution must display a null associated cost for a finite final time.

We first detail the case when $\alpha=0$ : system $\left(\Sigma_{1}\right)$ is just a double integrator. Let us now consider the following family of controls - see Figure 3:

$$
u_{\varepsilon, \Delta t}(t)=\left\{\begin{array}{cl}
\varepsilon & \text { for } t \in[0, \Delta t[ \\
0 & \text { for } t \in\left[\Delta t, T_{f}-\Delta t[\right. \\
-\varepsilon & \text { for } t \in\left[T_{f}-\Delta t, T_{f}\right]
\end{array}\right.
$$

The trajectory of $\left(\Sigma_{1}\right)$ is easily computed - see Figure 3 for an example. For a given $x_{f}$, we have:

$$
x\left(T_{f}\right)=\varepsilon \Delta t\left(T_{f}-\Delta t\right) \Rightarrow T_{f}=\frac{\varepsilon \Delta t^{2}+x_{f}}{\varepsilon \Delta t}
$$

The cost of this trajectory is $J(u)=\int_{0}^{\Delta t} \frac{\varepsilon y}{\rho(y)} d t+\int_{T_{f}-\Delta t}^{T_{f}} \frac{\varepsilon y}{\rho(y)} d t$ and tends toward 0 either when $\Delta t \rightarrow 0$ or $\varepsilon \rightarrow 0$. In both cases, $T_{f} \rightarrow \infty$ and the minimum cost is not reached. 

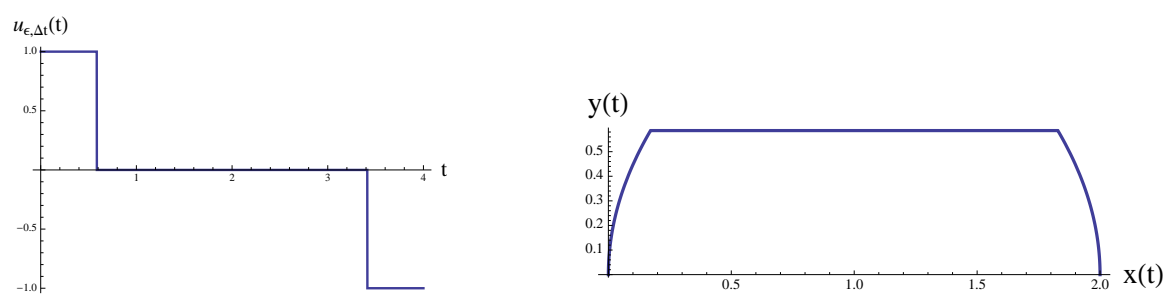

Figure 3: Control strategy of the form $u_{\varepsilon, \Delta t}(t)$, and associated trajectory.
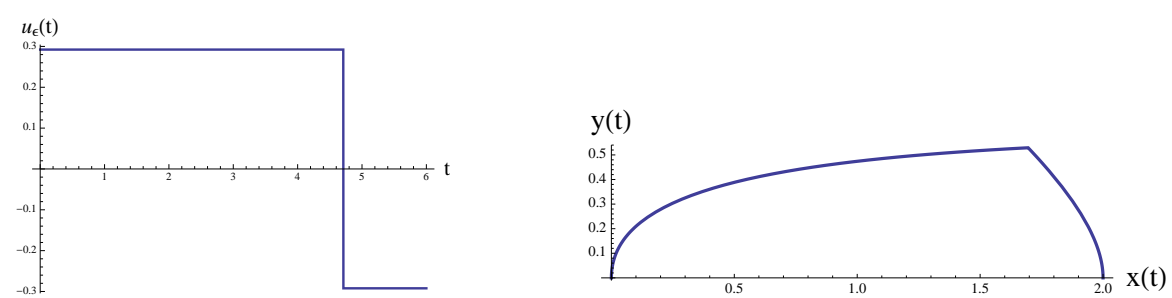

Figure 4: Control strategy of the form $u_{\varepsilon}(t)$, and associated trajectory.

For $\alpha>0$, we consider the family of controls:

$$
u_{\varepsilon}(t)=\left\{\begin{array}{cc}
\varepsilon & t \in[0, \Delta t[ \\
-\varepsilon & t \in\left[\Delta t, T_{f}\right]
\end{array}\right.
$$

Again, the trajectory is easily computed. For a fixed $\varepsilon>0$, the constraint $y\left(T_{f}\right)=0$ provide the expression $T_{f}=\frac{1}{\alpha} \ln \left(2 e^{\alpha \Delta t}-1\right)$. This latter expression is used together with the constraint $x\left(T_{f}\right)=x_{f}$ to provide the following expression for $\Delta t$ :

$$
\Delta t=\frac{1}{\alpha} \ln \left(e^{\frac{\alpha^{2} x_{f}}{\varepsilon}}+\sqrt{e^{\frac{\alpha^{2} x_{f}}{\varepsilon}}\left(e^{\frac{\alpha^{2} x_{f}}{\varepsilon}}-1\right)}\right)
$$

Since $\Delta t \stackrel{\varepsilon \rightarrow 0}{\longrightarrow} \infty$, the same goes for $T_{f}$ while $J\left(u_{\varepsilon}\right) \stackrel{\varepsilon \rightarrow 0}{\longrightarrow} 0$. As before, the minimum is not reached. An example of such a trajectory is shown in Figure 4.

\section{On the regularity of the problem}

In this section, we address two regularity issues:

- a theorem about the regularity of controls that are solution to a class of problems that includes $\left(\mathcal{P}_{1}\right)$;

- a lemma showing that for specific road profiles the search for optimal solutions can be limited to the set of forward trajectories.

\subsection{Regularity of the control variable}

Let $\left(\Sigma_{0}\right)$ be the following system for all $t \in[0, T]$ where $T>0$ is fixed:

$$
\left(\Sigma_{0}\right) \quad \dot{x}=F(x)+u G(x)
$$


The initial state of $\left(\Sigma_{0}\right)$ is denoted by $x_{0} \in \mathbb{R}^{n}, n>0$. The vector fields $F($.$) and G($.$) are$ assumed real analytic. The control variable is such that $|u(t)| \leq 1$. The associated input-output application, is:

$$
\begin{array}{rlrr}
\Phi: & \mathbb{R}^{n} \times L_{[0, T]}^{1} & \longrightarrow & \mathcal{A C}_{[0, T]} \\
& \left(x_{0}, u(t)\right) & \mapsto & x(.)
\end{array}
$$

where $x($.$) is the trajectory of \left(\Sigma_{0}\right)$ with initial state $x_{0}$ and control ${ }^{1} u($.$) , and \mathcal{A C}_{[0, T]}$ is the set of absolutely continuous functions over $[0, T]$. For a given $x_{0}$, we denote $\Phi_{x_{0}}(u)=\Phi\left(x_{0}, u\right)$. The set $L_{[0, T]}^{1}$ is embedded with its natural topology, $\mathcal{A C}_{[0, T]}$ has the topology of uniform convergence, and $\mathbb{R}^{n}$, the Euclidean metric topology.

Let $\psi($.$) be a continuous function. The cost associated to a trajectory of \left(\Sigma_{0}\right)$ generated by $u($.$) and x_{0}$ is:

$$
\begin{aligned}
J: \mathbb{R}^{n} \times L_{[0, T]}^{1} & \longrightarrow \mathbb{R}^{+} \\
\left(x_{0}, u(t)\right) & \mapsto \int_{0}^{T}|\psi(x) u| d t
\end{aligned}
$$

Lemma 1. $\Phi($.$) and J($.$) are uniformly continuous functions.$

Proof. For the sake of completeness, the proof of this lemma is given in Appendix A.

Theorem 1. Let $\mathcal{P} \mathcal{A}$ be the subset of $L_{[0, T]}^{1}$ made of piecewise algebraic controls on a set of full measure -i.e. almost everywhere algebraic on a finite number of subintervals of $[0, T]$. Let us consider an optimal control problem of the form:

$$
\left(\mathcal{P}_{0}\right)\left\{\begin{array}{cl}
\text { Minimise } & J(u)=\int_{0}^{T_{f}}|\psi(x(\tau)) u(\tau)| d \tau \\
\text { Subject to } & \left(\Sigma_{0}\right) \quad \dot{x}(t)=F(x(t))+u G(x(t)) \quad \text { for } t \in\left[0, T_{f}\right] \\
& x(0)=x_{0} \text { and } \quad x\left(T_{f}\right)=x_{f} \\
& |u(t)| \leq 1 \quad \text { for } t \in\left[0, T_{f}\right] \\
& T_{f}>0 \text { is fixed }
\end{array}\right.
$$

under the hypotheses:

$\left(\mathcal{H}_{0}\right) \psi($.$) has a maximum on \mathbb{R}^{n}$;

$\left(\mathcal{H}_{1}\right) J($.$) has a minimum on \mathcal{P} \mathcal{A}$, which we denote by $J_{M}$;

$\left(\mathcal{H}_{2}\right)$ the minimum time trajectories of $\left(\Sigma_{0}\right)$ are given by controls belonging to $\mathcal{P} \mathcal{A}$;

$\left(\mathcal{H}_{3}\right)\left(\Sigma_{0}\right)$ is small time locally controllable ${ }^{2}$ at $x_{f}$;

$\left(\mathcal{H}_{4}\right)\left(x_{f}, u_{f}\right)$ is an equilibrium point of $\left(\Sigma_{0}\right)$ such that $\psi\left(x_{f}\right) u_{f} \equiv 0$.

Then $J_{M}$ is the minimum of $J($.$) over L_{\left[0, T_{f}\right]}^{1}$.

The theorem is also true when $x_{f}$ is replaced by $x_{0}$ in hypotheses $\left(\mathcal{H}_{3}\right)$ and $\left(\mathcal{H}_{4}\right)$.

\footnotetext{
${ }^{1}$ Remind that, for a finite $T>0$, then $L_{[0, T]}^{\infty} \subset L_{[0, T]}^{1}$

${ }^{2} \mathrm{~A}$ control system $\left(\Sigma_{0}\right)$ is small time locally controllable from $x_{0}$, if for every positive time $T$ the set of points reachable from $x_{0}$ in time less than $T$ via admissible controls contains an open neighbourhood of $x_{0}-$ see e.g. [?].
} 
Proof. Let $x_{0}$ an initial state and $x_{f}$ be a final state. Let $u^{*} \in\left(L_{\left[0, T_{f}\right]}^{1} \backslash \mathcal{P} \mathcal{A}\right)$ be a control steering $\left(\Sigma_{0}\right)$ from $x_{0}$ to $x_{f}$ such that $J\left(u^{*}\right)<J_{M}$. We show that it contradicts $\left(\mathcal{H}_{1}\right)$.

Claim: for all $\varepsilon>0$, there exists an interval $I_{\varepsilon} \subset\left[0, T_{f}\right]$ of measure $\varepsilon$ such that $x^{*}$, the trajectory of $\left(\Sigma_{0}\right)$ given by $u^{*}$, is not the solution to the minimal time problem on $I_{\varepsilon}$.

Indeed, if this is not the case: there exists $\varepsilon>0$ such that, for all interval $I_{\varepsilon} \subset\left[0, T_{f}\right]$ of measure $\varepsilon, x^{*}$ is the solution to the minimal time problem on interval $I_{\varepsilon}$.

Therefore, according to $\left(\mathcal{H}_{2}\right)$, this means: there exists $\varepsilon>0$ such that, for all interval $I_{\varepsilon} \subset\left[0, T_{f}\right]$ of measure $\varepsilon$, the control $u^{*} \in \mathcal{P} \mathcal{A}$.

We deduce that $u^{*} \in \mathcal{P} \mathcal{A}$, which is in contradiction with the hypothesis: $u^{*} \notin \mathcal{P} \mathcal{A}$.

Let $u_{\varepsilon}$ be a new control constructed from $u^{*}$ as follows.

1. Let $t_{1}$ and $t_{2}$ denote the boundaries of $I_{\varepsilon}$, with $0 \leq t_{1}<t_{2} \leq T_{f}$.

2. For all $t \leq t_{1}, u_{\varepsilon}(t)=u^{*}(t)$.

3. Next, $u_{\varepsilon}(t)$ is equal to the solution to the minimum time problem associated to $\left(\Sigma_{0}\right)$ with starting point $x^{*}\left(t_{1}\right)$ and final point $x^{*}\left(t_{2}\right)$.

The corresponding travel time is denoted by $t_{\text {min }}$, and of course $t_{1}+t_{\text {min }}<t_{2}$.

Let $\delta$ denote the time saved in the process -i.e. $\delta=t_{2}-t_{1}-t_{\min }$.

4. Since $\left(\Sigma_{0}\right)$ is time invariant, for $\left.\left.t \in\right] t_{1}+t_{\text {min }}, T_{f}-\delta\right]$, then $u_{\varepsilon}(t)=u^{*}\left(t_{2}-t_{1}-t_{\text {min }}+t\right)$ steers $x$ from $x\left(t_{1}+t_{\text {min }}\right)=x\left(t_{2}\right)$ to $x_{f}$.

5. Finally, $u_{\varepsilon}(t)=u_{f}$ for $\left.\left.t \in\right] T_{f}-\delta, T_{f}\right]$.

The new control variable $u_{\varepsilon}$ steers $x$ from $x_{0}$ to $x_{f}$, and the cost difference between the trajectory associated to $u_{\epsilon}$ (denoted by $x_{\varepsilon}$ ) and the trajectory associated to $u^{*}$ is:

$$
\begin{aligned}
& J\left(u^{*}\right)-J\left(u_{\varepsilon}\right)=\int_{0}^{T_{f}}|\psi(x(t)) u(t)| d t-\int_{0}^{T_{f}}\left|\psi\left(x_{\varepsilon}(t)\right) u_{\varepsilon}(t)\right| d t \\
& =\underbrace{\int_{0}^{t_{1}}|\psi(x(t)) u(t)| d t}_{A}+\underbrace{\int_{t_{1}}^{t_{2}}|\psi(x(t)) u(t)| d t}_{B}+\underbrace{\int_{t_{1}}^{T_{f}}|\psi(x(t)) u(t)| d t}_{C} \\
& -\underbrace{\int_{0}^{t_{1}}\left|\psi\left(x_{\varepsilon}(t)\right) u_{\varepsilon}(t)\right| d t}_{T_{T_{f}-\delta}}-\underbrace{\int_{t_{1}}^{t_{1}+t_{\min }}\left|\psi\left(x_{\varepsilon}(t)\right) u_{\varepsilon}(t)\right| d t}_{E_{T_{f}}} \\
& -\underbrace{\int_{t_{1}+t_{\text {min }}}^{T_{f}-\delta}\left|\psi\left(x_{\varepsilon}(t)\right) u_{\varepsilon}(t)\right| d t}_{F}-\underbrace{\int_{T_{f}-\delta}^{T_{f}}\left|\psi\left(x_{\varepsilon}(t)\right) u_{\varepsilon}(t)\right| d t}_{G}
\end{aligned}
$$

1. By definition of $u_{\epsilon}$, we have $A=D$.

2. The measures of $\left[t_{1}+t_{\min }, T_{f}-\delta\right]$ and $\left[t_{2}, T_{f}\right]$ are the same $\left(T_{f}-\delta-t_{1}-t_{\min }=T_{f}-t_{2}\right)$. Furthermore

(a) for $t \in\left[0, T_{f}-t_{2}\right], u_{\varepsilon}\left(t_{1}+t_{\text {min }}+t\right)=u^{*}\left(t_{2}+t\right)$, 
(b) $x_{\varepsilon}\left(t_{1}+t_{\text {min }}\right)=x^{*}\left(t_{2}\right)$,

(c) $\left(\Sigma_{0}\right)$ is time invariant.

Thus, $\forall t \in\left[0, T_{f}-t_{2}\right], x_{\varepsilon}\left(t_{1}+t_{\text {min }}+t\right)=x^{*}\left(t_{2}+t\right)$.

And finally, since the function $\psi($.$) is time invariant, then C=F$.

3. since $\left(x_{\varepsilon}, u_{\varepsilon}\right)=\left(x_{f}, u_{f}\right)$ over $\left[T_{f}-\delta, T_{f}\right]$, then $G=0$-cf. hypothesis $\left(\mathcal{H}_{4}\right)$.

Those considerations lead to:

$$
\begin{aligned}
\left|J\left(u^{*}\right)-J\left(u_{\varepsilon}\right)\right| & =\left|\int_{t_{1}}^{t_{2}}\right| \psi(x(t)) u(t)\left|d t-\int_{t_{1}}^{t_{1}+t_{\text {min }}}\right| \psi\left(x_{\varepsilon}(t)\right) u_{\varepsilon}(t)|d t| \\
& \leq \int_{t_{1}}^{t_{1}} \underbrace{|\psi(x(t))|}_{\leq M} \underbrace{|u(t)|}_{\leq 1} d t+\int_{t_{1}}^{t+t_{\text {min }}}\left|\psi\left(x_{\varepsilon}(t)\right)\right|\left|u_{\varepsilon}(t)\right| d t \\
& \leq M\left(t_{2}-t_{1}\right)+M t_{\min } \leq 2 \varepsilon M
\end{aligned}
$$

where $M>0$ denotes the maximum of $\psi($.$) -cf. hypothesis \left(\mathcal{H}_{0}\right)$.

Henceforth, we fix $\varepsilon>0$ such that $2 \varepsilon M<\frac{J_{M}-J\left(u^{*}\right)}{3}$, and $x_{\varepsilon}$ is the trajectory of $\left(\Sigma_{0}\right)$ given by $x_{0}$ and $u_{\varepsilon}$.

As a consequence, the small time $\delta>0$ is now fixed. The same goes for the accessibility set of $\left(-\Sigma_{0}\right)$ starting $^{3}$ from $x_{f}$ for times less or equal than $\delta$. We denote this set by $\mathcal{A}_{\delta}^{-}\left(x_{f}\right)$.

Following [?]:

1. the small time local controllability of $\left(\Sigma_{0}\right)$, implies the small time local controllability of $\left(-\Sigma_{0}\right)$;

2. the real analyticity hypothesis for $F(x)$ and $G(x)$ leads to the small time self-reachability of $\left(-\Sigma_{0}\right)$;

3. since $x_{f}$ is an equilibrium point of $\left(-\Sigma_{0}\right)$, then self-reachability is valid in fixed time.

Therefore, for any point in $\mathcal{A}_{\delta}^{-}\left(x_{f}\right)$, there exists a control $u_{e} \in \mathcal{P} \mathcal{A}$ steering this point to $x_{f}$ in a time exactly equal to $\delta$. The cost of such a piece of trajectory is:

$$
\int_{T_{f}-\delta}^{T_{f}}\left|\psi\left(x_{e}(t)\right) u_{e}(t)\right| d t \leq 2 \delta M<2 \varepsilon M<\frac{J_{M}-J\left(u^{*}\right)}{3}
$$

Let us now construct a $L_{\left[0, T_{f}\right]}^{1}$-perturbation of $u_{\varepsilon}$ that lives in $\mathcal{P} \mathcal{A}$ and denoted by $u_{\delta}$. It is important to point out that the reachable set $\mathcal{A}_{\delta}^{-}\left(x_{f}\right)$ is now independent from the construction that follows. This is a crucial point of the proof.

Firstly, following Luzin's theorem [14], for all $m>0$ there is a continuous function $\mathcal{C}_{\delta}$ such that $\left\{t: \mathcal{C}_{\delta}(t) \neq u_{\varepsilon}(t)\right\}$ is of measure at most $m$. Next, the function $\mathcal{C}_{\delta}$ is saturated in \pm 1 in order to obtain a continuous function $\overline{\mathcal{C}}_{\delta}$ such that $\left\|u_{\varepsilon}-\overline{\mathcal{C}}_{\delta}\right\|_{L^{1}}<2 m$.

Secondly, there is a sequence of polynomials, denoted by $\left\{P_{n}\right\}_{n \in \mathbb{N}}$, that converges uniformly to $\overline{\mathcal{C}}_{\delta}$. Therefore, for all $\tilde{m}>0$, there exists a polynomial $P_{\delta}$ taken from $\left\{P_{n}\right\}_{n \in \mathbb{N}}$ which is

\footnotetext{
${ }^{3}\left(-\Sigma_{0}\right)$ denotes system $\left(\Sigma_{0}\right)$ with reversed time.
} 
$\tilde{m}$-close to $\overline{\mathcal{C}}_{\delta}$ in the $L_{\left[0, T_{f}\right]}^{1}$-sense. We consider the control variable $u_{\delta}(t) \in \mathcal{P} \mathcal{A}$ obtained from the polynomial $P_{\delta}$ saturated in \pm 1 , then $\left\|u_{\varepsilon}-u_{\delta}\right\|_{L^{1}}<2 m+\tilde{m}$.

The uniform continuity of $\Phi_{x_{0}}($.$) , and J_{x_{0}}($.$) , cf. Lemma 1$, guarantees that we can choose $m$ and $\tilde{m}$ such that:

1. $\left|J\left(u_{\varepsilon}\right)-J\left(u_{\delta}\right)\right|<\frac{J_{M}-J\left(u^{*}\right)}{3}$,

2. $x_{\delta}\left(T_{f}-\delta\right)$ is close enough to $x_{f}$, that is to say $x_{\delta}\left(T_{f}\right) \in \mathcal{A}_{\delta}^{-}\left(x_{f}\right)$.

Finally, the following control $\tilde{u} \in \mathcal{P} \mathcal{A}$ steers $x_{0}$ to $x_{f}$ in a fixed time $T_{f}$ :

$$
\tilde{u}(t)= \begin{cases}u_{\delta}(t) & \text { if } 0 \leq t \leq T_{f}-\delta \\ u_{e}(t) & \text { otherwise }\end{cases}
$$

In particular, we have the relations $J(\tilde{u})=J\left(u_{\delta}\right)+J\left(u_{e}\right)$ and

$$
\left|J\left(u^{*}\right)-J(\tilde{u})\right|<\underbrace{\left|J\left(u^{*}\right)-J\left(u_{\varepsilon}\right)\right|}_{<\frac{J_{M}-J\left(u^{*}\right)}{3}}+\underbrace{\left|J\left(u_{\varepsilon}\right)-J\left(u_{\delta}\right)\right|}_{<\frac{J_{M}-J\left(u^{*}\right)}{3}}+\underbrace{\left|J\left(u_{e}\right)\right|}_{<\frac{J_{M}-J\left(u^{*}\right)}{3}}<J_{M}-J\left(u^{*}\right)
$$

which contradicts the minimality of $J_{M}$ over the subset $\mathcal{P} \mathcal{A}$ - i.e. hypothesis $\left(\mathcal{H}_{1}\right)$ - and concludes the proof.

Now, let us show that this result is preserved when $\left(x_{0}, u_{0}\right)$ is the equilibrium point around which $\left(\Sigma_{0}\right)$ is small time locally controllable.

- The control $u_{\varepsilon}(t)$ is constructed in the same way as before, except that it equals $u_{0}$ over $[0, \delta]$.

- The small time $\delta$ being fixed during the previous step, the same goes for the reachable set of $\left(\Sigma_{0}\right)$ starting from $x_{0}$, in time less or equal to $\delta: \mathcal{A}_{\delta}^{+}\left(x_{0}\right)$.

As before, the results on small time self-reachability guarantee the existence of a control on $\mathcal{P} \mathcal{A}$ that steers $x_{0}$ to any point of $\mathcal{A}_{\delta}^{+}\left(x_{0}\right)$ in a time that is exactly equal to $\delta$. The cost of such a piece of trajectory is less than $\frac{J_{M}-J\left(u^{*}\right)}{3}$.

- $x_{\varepsilon}(t)$ denotes the trajectory starting from $x_{0}$ when the control $u_{\varepsilon}$ is applied. We define $\tilde{x}_{\varepsilon}(\tau)=x_{\varepsilon}\left(T_{f}-\tau\right)$ which dynamics are:

$$
\frac{d \tilde{x}_{\varepsilon}}{d \tau}=-\left.\frac{x_{\varepsilon}(t)}{d t}\right|_{\left(T_{f}-\tau\right)}=-F\left(\tilde{x}_{\varepsilon}(\tau)\right)-u_{\varepsilon}\left(T_{f}-\tau\right) G\left(\tilde{x}_{\varepsilon}(\tau)\right)
$$

The control $\tilde{u}_{\varepsilon}(\tau)=u_{\varepsilon}\left(T_{f}-\tau\right)$, for $\tau \in\left[0, T_{f}-\delta\right]$, applied to $\left(-\Sigma_{0}\right)$ steers $\tilde{x}(0)=x_{f}$ to $\tilde{x}\left(T_{f}-\delta\right)=x_{0}$.

Again, we use Luzin's theorem and the density property of polynomials. Since Lemma 1 also applies to system $\left(-\Sigma_{0}\right)$, there is therefore a control $\tilde{u}_{\delta} \in \mathcal{P} \mathcal{A}$ such that $\left|J\left(\tilde{u}_{\delta}\right)-J\left(\tilde{u}_{\varepsilon}\right)\right|<$ $\frac{J_{M}-J\left(u^{*}\right)}{3}$, such that $\tilde{x}_{\delta}\left(T_{f}-\delta\right) \in \mathcal{A}_{\delta}^{+}\left(x_{0}\right)$.

- With a change of variables similar to the one of the previous step -i.e. $x_{\delta}(t)=\tilde{x}_{\delta}\left(T_{f}-t\right)$ the control $u_{\delta}(t)=\tilde{u}_{\delta}\left(T_{f}-t\right)$, for $t \in\left[\delta, T_{f}\right]$, applied to $\left(\Sigma_{0}\right)$, steers some point $x_{\delta}(\delta) \in$ $\mathcal{A}_{\delta}^{+}\left(x_{0}\right)$ to $x_{\delta}\left(T_{f}\right)=\tilde{x}_{\delta}(0)=x_{f}$.

- Finally, a $\mathcal{P} \mathcal{A}$ control steering $x_{0}$ to $x_{f}$, with an associated cost less than $J_{M}$ is obtained by firstly joining $x_{0}$ to $x_{\delta}(\delta) \in \mathcal{A}_{\delta}^{+}\left(x_{0}\right)$ for $t \in[0, \delta]$, and secondly using $u_{\delta}(t)$ for $t \in\left[\delta, T_{f}\right]$.

Remark 2. The small time self-reachability of $\left(\Sigma_{0}\right)$ is a key point of the proof. As it can be seen in Corollary 4.15 of [?], the property " $F($.$) and G($.$) are real analytic vector fields" can be$ replaced by " $F($.$) and G($.$) are \mathcal{C}^{1}$ and locally bounded". 


\subsection{Trajectories without backward arcs}

A backward arc is understood as a piece of trajectory, defined over a non-trivial interval of time, along which the quantity $y$ is strictly negative.

Lemma 2. Let us consider a road for which the profile is such that Problem $\left(\mathcal{P}_{1}\right)$ is invariant under a translation along the $x$-axis of the phase diagram of $\left(\Sigma_{1}\right)$. Then, trajectories including backward arcs can be disregarded when looking for solutions to $\left(\mathcal{P}_{1}\right)$.

Proof. Let $\Gamma(t)=\left(x_{\Gamma}(t), y_{\Gamma}(t)\right)^{t}$ be a trajectory of $\left(\Sigma_{1}\right)$. According to Theorem 1 , in order to be a candidate for optimality, this trajectory is made of a finite number of arcs. Let us assume that $y_{\Gamma}(t)$ has only one backward arc - the following argument remains in the case of multiple backward arcs:

$$
y_{\Gamma}(t)=\left\{\begin{array}{l}
y_{\Gamma}(t)<0 \text { over }\left[0, t_{1}[\right. \\
y_{\Gamma}(t) \geq 0 \text { over }\left[t_{1}, T_{f}\right]
\end{array}\right.
$$

Let $\Gamma^{-}$denote the arc such that $y<0$, and $\Gamma^{+}$the other one. We now construct a new trajectory, denoted by $\gamma=\left(x_{\gamma}, y_{\gamma}\right)^{t}$, that doesn't include any backward arc and having an associated cost less or equal to the one of $\Gamma$. The explanations below are illustrated in Figure 5.

Let us translate the arc $\Gamma^{+}$along the $x$-axis in order to move $A=\left(x_{\Gamma}\left(t_{1}\right), y_{\Gamma}\left(t_{1}\right)\right)^{t}$ to $(0,0)^{t}$. This new curve, denoted by $\Gamma_{\text {trans }}^{+}$is the dotted curve in Figure 5 . The time instants $t_{2}$ and $t_{3}$ are defined as follows:

- let $B$ be the intersection point between $\Gamma^{+}$and $\Gamma_{\text {trans }}^{+}$, then $B=\left(x_{\Gamma}\left(t_{3}\right), y_{\Gamma}\left(t_{3}\right)\right)^{t}$;

- let $C$ be the translation of $B$ when $\Gamma_{\text {trans }}^{+}$is translated back to $\Gamma^{+}$, then $C=\left(x_{\Gamma}\left(t_{2}\right), y_{\Gamma}\left(t_{2}\right)\right)^{t}$.

The trajectory $\gamma$ is:

$$
\gamma(t)=\left\{\begin{aligned}
\Gamma_{\text {trans }}^{+}\left(t+t_{1}\right) & \text { for } 0 \leq t \leq t_{2}-t_{1} \\
\Gamma^{+}\left(t+t_{3}\right) & \text { for } t_{2}-t_{1} \leq t \leq\left(t_{2}-t_{1}\right)+\left(T_{f}-t_{3}\right) \\
\left(x_{f}, 0\right)^{t} & \text { for }\left(t_{2}-t_{1}\right)+\left(T_{f}-t_{3}\right) \leq t \leq T_{f}
\end{aligned}\right.
$$

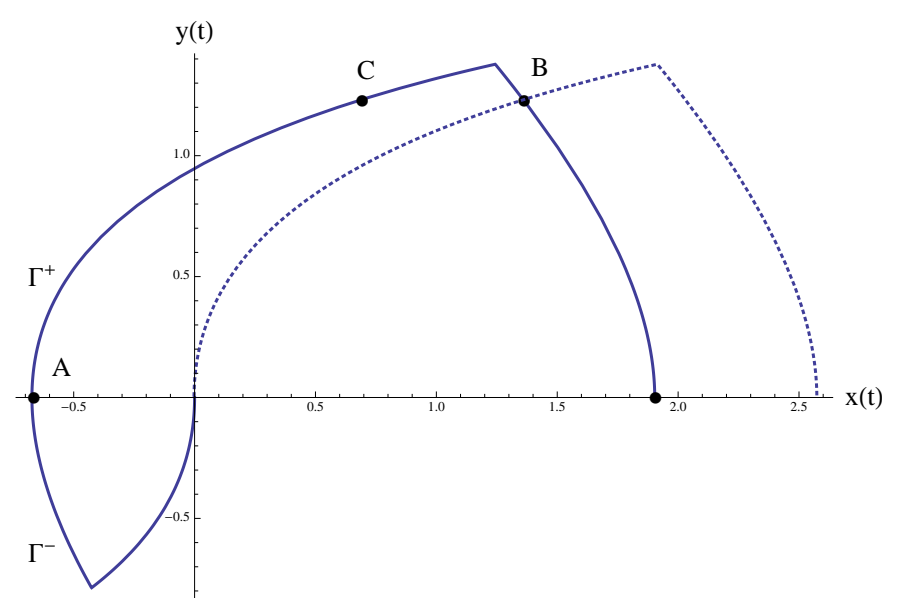

Figure 5: The $\Gamma(t)$ trajectory. 
Let $u_{\Gamma}$ (resp. $\left.u_{\gamma}\right)$ denote the control corresponding to $\Gamma($ resp. $\gamma)$, and $J^{\Gamma}(u)\left(\right.$ resp. $J^{\gamma}(u)$ ), then:

$$
\begin{aligned}
J^{\Gamma}(u) & =\int_{0}^{T_{f}} \frac{\left|y_{\Gamma} u_{\Gamma}\right|}{\rho\left(y_{\Gamma}\right)} d t=\int_{0}^{t_{1}} \frac{\left|y_{\Gamma} u_{\Gamma}\right|}{\rho\left(y_{\Gamma}\right)} d t+\int_{t_{1}}^{t_{2}} \frac{\left|y_{\Gamma} u_{\Gamma}\right|}{\rho\left(y_{\Gamma}\right)} d t+\int_{t_{2}}^{t_{3}} \frac{\left|y_{\Gamma} u_{\Gamma}\right|}{\rho\left(y_{\Gamma}\right)} d t+\int_{t_{3}}^{T_{f}} \frac{\left|y_{\Gamma} u_{\Gamma}\right|}{\rho\left(y_{\Gamma}\right)} d t \\
& \geq \int_{t_{1}}^{t_{2}} \frac{\left|y_{\Gamma} u_{\Gamma}\right|}{\rho\left(y_{\Gamma}\right)} d t+\int_{t_{3}}^{T_{f}} \frac{\left|y_{\Gamma} u_{\Gamma}\right|}{\rho\left(y_{\Gamma}\right)} d t=\int_{0}^{t_{2}-t_{1}} \frac{\left|y_{\gamma} u_{\gamma}\right|}{\rho\left(y_{\gamma}\right)} d t+\int_{t_{2}-t_{1}}^{\left(t_{2}-t_{1}\right)+\left(T_{f}-t_{3}\right)} \frac{\left|y_{\gamma} u_{\gamma}\right|}{\rho\left(y_{\gamma}\right)} d t=J^{\gamma}(u)
\end{aligned}
$$

\section{Case studies}

In this paper, three configurations of Problem $\left(\mathcal{P}_{1}\right)$ are investigated:

1. a flat road without drag;

2. a flat road with drag;

3. a non-flat road profile, and a null drag coefficient.

System $\left(\Sigma_{1}\right)$ is linear in the two first configurations, and nonlinear in the third. As we deal with a fixed time control problem, a solution exists only if the final time $T_{f}$ is larger than the minimum time $\left(T_{\min }\right)$ needed to reach the prescribed destination. In all those three cases, the minimum time strategy consists of the following control sequence, and the calculation of $T_{\min }$ is straightforward:

$$
u_{\min }(t)=\left\{\begin{array}{l}
1 \text { for } 0 \leq t<\Delta t \\
-1 \text { for } \Delta t \leq T_{\min }
\end{array}\right.
$$

Case (1) is an elementary example treated in the first chapter of [17], and cases (2) and (3) are easily dealt with following the procedure in [17]. The expressions of the minimum travel time for cases (1) and (2) are, respectively, the two expressions below:

$$
T_{\min }=2 \sqrt{x_{f}} \quad \text { and } \quad T_{\min }=\frac{1}{\alpha}\left(\ln \left(1+\sqrt{1-e^{-\alpha^{2} x_{f}}}\right)-\ln \left(1-\sqrt{1-e^{-\alpha^{2} x_{f}}}\right)\right)
$$

In the last case, the formula is provided in Section 4.3 below.

The study is performed with the help of Pontryagin's Maximum Principle [17, 1, 10, 7]. To this end, we introduce $\mathcal{P}(t)=(p(t), q(t))^{t}$, the adjoint vector of $X(t)=(x(t), y(t))^{t}$ and form the Hamiltonian:

$$
\mathcal{H}\left(X, \mathcal{P}, u, \lambda_{0}\right)=p y+q(u-\alpha y-\sin (\theta(x)))-\lambda_{0} \frac{|y u|}{\rho(y)} \quad \text { with } \lambda_{0} \in\{1,0\}
$$

Since $\left(\mathcal{P}_{1}\right)$ is an autonomous problem, then $\mathcal{H}\left(X, \mathcal{P}, u, \lambda_{0}\right)$ is constant along extremal trajectories and denoted by $H$. Also, still along extremal trajectories, for almost every t, we have the maximisation condition:

$$
H=\max _{|u| \leq 1} \mathcal{H}\left(X, \mathcal{P}, u, \lambda_{0}\right)
$$

and the adjoint equations:

$$
\begin{aligned}
\dot{p} & =-\frac{\partial \mathcal{H}}{\partial x}\left(X, \mathcal{P}, u, \lambda_{0}\right) \\
-\dot{q} & \in D_{y} \mathcal{H}\left(X, \mathcal{P}, u, \lambda_{0}\right)
\end{aligned}
$$


where $D_{y} \mathcal{H}$ denotes the subdifferential of $\mathcal{H}$ with respect to $y$, cf. [10].

As a consequence, following the maximisation condition, the candidate control strategy for abnormal extremals is given by:

$$
u(t)=\underset{|u| \leq 1}{\operatorname{argmax}} \mathcal{H}(X, \mathcal{P}, u, 0)=\operatorname{sgn}(q)
$$

For normal extremals the maximisation condition, illustrated in Figure 6, yields:

$$
u(t)=\underset{|u| \leq 1}{\operatorname{argmax}} \mathcal{H}(X, \mathcal{P}, u, 1)=\underset{|u| \leq 1}{\operatorname{argmax}}\left(q u-\frac{|y u|}{\rho(y)}\right)= \begin{cases}1 & \text { if } q>\frac{|y|}{\rho(y)} \\ u \in[0,1] & \text { if } q=\frac{|y|}{\rho(y)} \\ 0 & \text { if }-\frac{|y|}{\rho(y)}<q<\frac{|y|}{\rho(y)} \\ u \in[-1,0] & \text { if } q=-\frac{|y|}{\rho(y)} \\ -1 & \text { if } q<-\frac{|y|}{\rho(y)}\end{cases}
$$

A singular arc is a piece of extremal trajectory such that either $q=\frac{|y|}{\rho(y)}$ or $q=-\frac{|y|}{\rho(y)}$ over a non-trivial interval of time - see for instance [7,6, 20]. Also, a control $u(t)$ is said extremal if there exists $\mathcal{P}(0)=\left(p_{0}, q_{0}\right)$ such that the trajectories of the Hamiltonian system, formed by $\left(\Sigma_{1}\right),(9)$ and (10) is extremal.

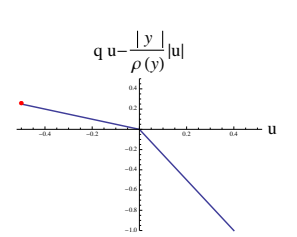

(a) $q<-\frac{|y|}{\rho(y)}$

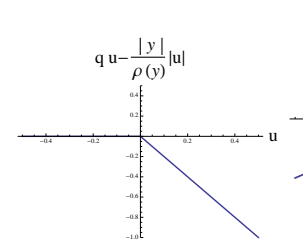

(b) $q=-\frac{|y|}{\rho(y)}$

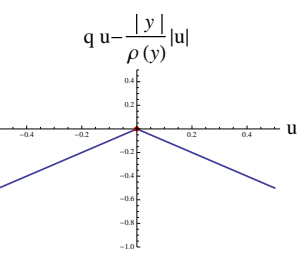

(c) $-\frac{|y|}{\rho(y)}<q<\frac{|y|}{\rho(y)}$

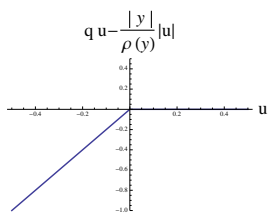

(d) $q=\frac{|y|}{\rho(y)}$

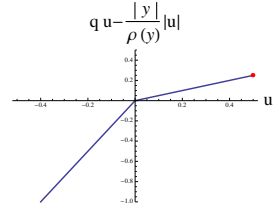

(e) $\frac{|y|}{\rho(y)}<q$

Figure 6: The maximisation condition in the normal case.

\section{On the properties of singular arcs.}

Let us write the Hamiltonian along an extremal singular arc. Following the set of rules (11), we have:

$$
H=p y-q(\sin (\theta(x))+\alpha y) \text { where } q(t) \text { is either } \frac{|y(t)|}{\rho(y(t))} \text { or }-\frac{|y(t)|}{\rho(y(t))}
$$

As a consequence, if the $y$ trajectory of an extremal singular arc crosses $y=0$, then $H=0$ along the corresponding extremal.

We now consider the case $q=\frac{|y|}{\rho(y)}$ and $y>0$, then $u \in[0,1]$ and the Hamiltonian is

$$
\mathcal{H}\left(X, \mathcal{P}, u, \lambda_{0}\right)=p y-\frac{y}{\rho(y)}(\sin (\theta(x))+\alpha y)
$$


Its time derivative is:

$$
0=\dot{y}\left[p-\sin (\theta(x))\left(\frac{1}{\rho(y)}-\frac{y \rho^{\prime}(y)}{\rho^{2}(y)}\right)-\alpha y\left(\frac{2}{\rho(y)}-\frac{y \rho^{\prime}(y)}{\rho^{2}(y)}\right)\right] \text { where } \rho^{\prime}(y)=\frac{\partial \rho(y)}{\partial y}
$$

This last expression is of the form $0=\dot{y}[p-\sin (\theta(x)) A-B]$. Therefore, one possible behaviour for singular arcs is to have $y$ constant. Over intervals such that $y$ is not constant, the quantity $[p-\sin (\theta(x)) A-B]$ must vanish. Following equation (9), the time derivative of $p$ is given by:

$$
\dot{p}=q \cos (\theta(x)) \frac{\partial \theta(x)}{\partial x}=\frac{y}{\rho(y)} \cos (\theta(x)) \frac{\partial \theta(x)}{\partial x}
$$

If $\frac{\partial \theta}{\partial x}$ doesn't vanish, the time derivative of $[p-\sin (\theta(x)) A-B]$ is of the form: $C+\dot{y} D=0$ where ${ }^{4}$ both $C$ and $D$ are independent from $\dot{y}$. At this point one can investigate possible behaviours for extremal singular arcs by using the differential equation $\dot{y}=-\frac{C}{D}$.

If $\frac{\partial \theta}{\partial x}$ vanishes, then both $p$ and $\sin (\theta(x))$ are constants. The time derivative of $[p-\sin (\theta(x)) A-B]$ is of the form $(\dot{y} D)$ where $D$ depends on $y, \rho(y), \rho^{\prime}(y)$ and $\rho^{\prime \prime}(y)$. Since we assumed that $\dot{y} \neq 0$, then $D=0$, and the time derivative of $D$ is computed. It is of the form $(\dot{y} E)$ where $E$ depends on $y, \rho(y)$, and the derivatives of $\rho$ up to order 3. In fact, there is no new information to be found and an extremal singular arc of this kind exists only if the yield function is of the form given by the Hamiltonian (12), which is almost never the case.

The same conclusions are drawn for $y<0$ and in the case $q=-\frac{|y|}{\rho(y)}$.

\subsection{Flat road without drag}

In this section, the drag coefficient $\alpha$ is null and the road is flat -i.e. $\theta(x) \equiv 0$. System $\left(\Sigma_{1}\right)$ is then a simple double integrator and the Hamiltonian associated to Problem $\left(\mathcal{P}_{1}\right)$ is:

$$
\mathcal{H}\left(X, \mathcal{P}, u, \lambda_{0}\right)=p y+q u-\lambda_{0} \frac{|y u|}{\rho(y)}
$$

System $\left(\Sigma_{1}\right)$ being linear, there is no abnormal extremal trajectory. Let us write the Hamiltonian, along extremal trajectories at times $t=0$ and $t=T_{f}$ :

$$
\mathcal{H}_{\mid t=0}=\mathcal{H}_{\mid t=T_{f}}=H \Rightarrow H=u(0) q(0)=u\left(T_{f}\right) q\left(T_{f}\right)
$$

From Lemma $2, y \geq 0$ for all $t \in\left[0, T_{f}\right]$. There exist $t_{1}$ and $t_{2}$ in $\left[0, T_{f}\right]$ such that $y\left(t_{1}\right)=y\left(t_{2}\right)=0$ and $y(t)>0$ for all $t \in] t_{1}, t_{2}\left[\right.$. The quantity $\kappa(t)=\frac{q \rho(y)}{y}$ is well defined over $] t_{1}, t_{2}[$ and the set of rules (11) translates into:

$$
u(t)= \begin{cases}1 & \text { if } \quad \kappa(1)>1 \\ 0 & \text { if }-1<\kappa(t)<1 \\ -1 & \text { if } \kappa(t)<-1\end{cases}
$$

Since there is no backward arc, there exists $\varepsilon>0$ such that $u=1$ over an interval of the form ]$t_{1}, t_{1}+\varepsilon\left[\right.$. Remember that $\left(\Sigma_{1}\right)$ is a double integrator and that $y\left(t_{2}\right)=0$. This means that the control variable must become negative at some point (or equivalently, $\kappa$ must be less than -1 ),

\footnotetext{
${ }^{4}$ The expressions $C$ and $D$ are rather long, and as such, we do not reproduce them in the paper. It is straightforward to recover them with the help of a symbolic computation software. They can also be found in [?].
} 
thus there is a time $\left.t^{\star} \in\right] t_{1}, t_{2}$ [ such that $\kappa\left(t^{\star}\right)=0$ and $\dot{\kappa}<0$. Note that, $\kappa\left(t^{\star}\right)=0$ cannot be an inflexion point as it is shown later on. First, let us compute the time derivative of $\kappa(t)$ :

$$
\begin{aligned}
\dot{\kappa}(t) & =\left(\widehat{\frac{q(y)}{y}}\right)=\frac{q \dot{y} y \rho^{\prime}(y)}{y^{2}}+\frac{\dot{q} \rho(y) y}{y^{2}}-\frac{q \rho(y) \dot{y}}{y^{2}} \\
& =\frac{q \dot{y} \rho^{\prime}(y)}{y}+\rho(y) \frac{1}{y^{2}} \underbrace{(\dot{q} y-q \dot{y})}_{-H-\frac{|u| \rho^{\prime}(y) y^{2}}{\rho(y)^{2}}}=-\rho(y) \frac{H}{y^{2}}+\rho^{\prime}(y)\left(\frac{q \dot{y}}{y}-\frac{|u|}{\rho(y)}\right) \\
& =-\frac{\rho(y)}{y^{2}} H+\frac{\rho^{\prime}(y)}{\rho(y)}(\kappa u-|u|)
\end{aligned}
$$

Following (14), $u=0$ when $\kappa \in]-1,1\left[\right.$. Therefore, equation (15) yields $\dot{\kappa}\left(t^{\star}\right)=-\frac{\rho(y)}{y^{2}} H$. If $\kappa\left(t^{\star}\right)=0$ is an inflexion point, then $H=0$ along the extremal trajectory. As a consequence, from $(15), \dot{\kappa}=0$ as soon as $u=0$ and $\kappa$ never reaches -1 . Therefore, $\kappa\left(t^{\star}\right)=0$ is not an inflexion point, and $H$ must be strictly positive.

Now, relation (13) tells us that $u(0)=1$ and $u\left(T_{f}\right)=-1$. Indeed, since $u(0) q(0) \neq 0$ then $q(0) \neq 0$. If $q(0)<0$ then $u(0)=-1$ which contradicts the fact that there is no backward arc. A similar argument yields $u\left(T_{f}\right)=-1$.

Going back to equation (15), let us consider the case when $\kappa=1$. According to the set of rules $(14), u \geq 0$, therefore $(\kappa u-|u|)=0$ and $\dot{\kappa}=-\frac{\rho(y)}{y^{2}} H<0$. When $\kappa=-1, u \leq 0$ and $\dot{\kappa}$ is also strictly negative. As a consequence, an extremal trajectory contains no singular arc and has a maximum of 2 commutations. In fact, for a given $T_{f}>T_{m i n}$, there is only one possible extremal trajectory, the one generated by the control sequence:

$$
u(t)= \begin{cases}1 & \text { for } t \in[0, \Delta t[ \\ 0 & \text { for } t \in\left[\Delta t, T_{f}-\Delta t[\right. \\ -1 & \text { for } t \in\left[T_{f}-\Delta t, T_{f}\right]\end{cases}
$$

The terminal condition $x_{f}=\Delta t T_{f}-\Delta t^{2}$ allows the explicit computation of $\Delta t$ :

$$
\Delta t=\frac{1}{2}\left(T_{f}-\sqrt{T_{f}^{2}-4 x_{f}}\right)
$$

This relation is well defined. Indeed, $T_{f}>T_{\min }$ implies $\left(T_{f}^{2}-4 x_{f}\right)>0$-cf. equation (8).

It is clear from expression (15), that the yield function has no part to play in this specific problem. Actually, the solution to this problem is exactly the same as the one to the problem that consists of minimising $\int_{0}^{T_{f}}|u(t)| d t$ for a double integrator. The interested reader can refer to [18] where this problem is solved.

Figure 7 illustrates this optimal strategy. The trajectory is shown in Figure 7a. Figures $7 \mathrm{~b}$ and $7 \mathrm{~d}$ show the switching functions for a constant and a non-constant yield function respectively. Finally, Figure 7c represents the non-constant yield function: the dotted part shows the shape of the yield function, while the plain red part shows the visited values of the yield function. 


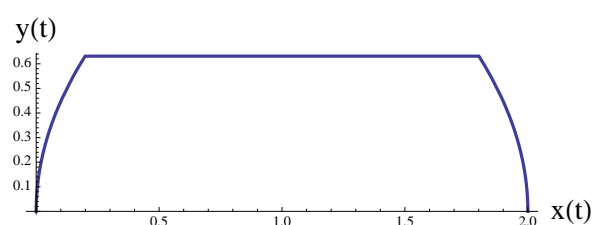

(a)

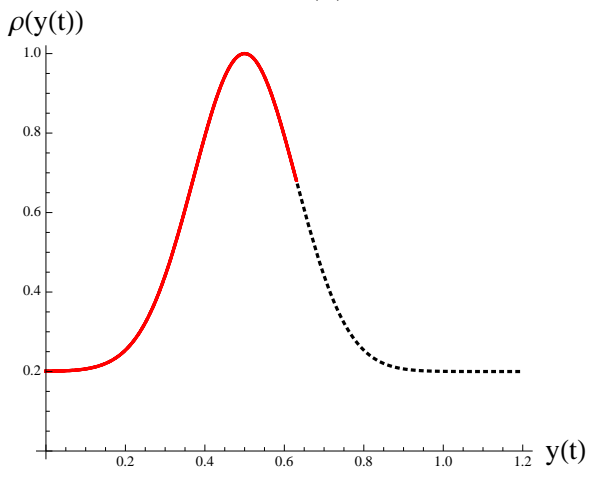

(c)

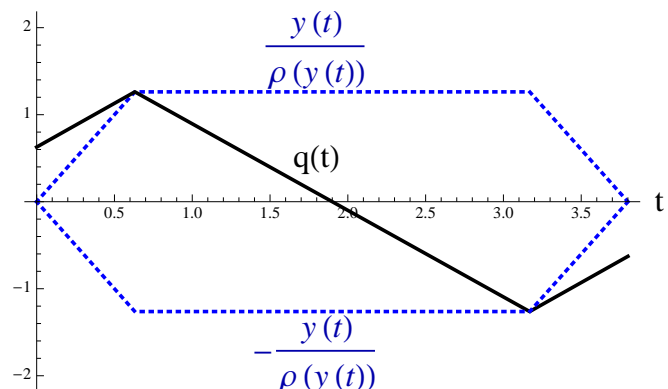

(b)

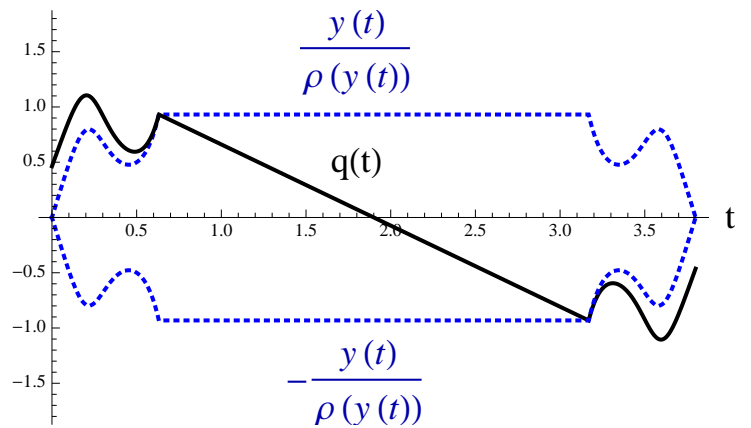

(d)

Figure 7: Optimal trajectory in the double integrator case. Parameters: $x_{f}=2, T_{\min } \approx 2.82$ and $T_{f}=3.8$. 


\subsection{Flat road with drag}

In this second case study a drag coefficient is introduced. Again, system $\left(\Sigma_{1}\right)$ is linear and is written:

$$
\left\{\begin{array}{l}
\dot{x}=y \\
\dot{y}=u-\alpha y
\end{array}\right.
$$

As before, abnormal extremal trajectories are out of the picture, Lemma 2 applies and optimal trajectories don't include backward arcs. The normal Hamiltonian $\mathcal{H}$ is:

$$
\mathcal{H}(X, \mathcal{P}, u, 1)=p y+q(u-\alpha y)-\frac{y|u|}{\rho(y)}
$$

As in Section 4.1, we have the relation: $H=u(0) q(0)=u\left(T_{f}\right) q\left(T_{f}\right)$, and we define the quantity $\kappa=\frac{q \rho(y)}{y}$. It is defined over some open subinterval $] t_{1}, t_{2}\left[\subset\left[0, T_{f}\right]\right.$, such that $y(t)>0$ and $y\left(t_{1}\right)=y\left(t_{2}\right)=0$. The time derivative of $\kappa$ is:

$$
\dot{\kappa}(t)=-\frac{\rho(y)}{y^{2}} H-\frac{\rho^{\prime}(y)}{\rho(y)}|u|+\kappa(t)\left(\alpha+\frac{\rho^{\prime}(y)}{\rho(y)}(u-\alpha y)\right)
$$

As in section 4.1 , there exists $\varepsilon>0$ such that $u=1$ over an interval of the form $] t_{1}, t_{1}+\varepsilon[$, and $\left(\Sigma_{1}\right)$ is such that the control $u$ must be negative in order to have $y\left(T_{f}\right)=0$. As a consequence, there is a time $t^{\star}$ such that $\kappa\left(t^{\star}\right)$ vanishes, and $\dot{\kappa}\left(t^{\star}\right)<0$.

Again, $\kappa\left(t^{\star}\right)=0$ cannot be an inflexion point. Indeed, according to (17), if $\kappa\left(t^{\star}\right)=0$ is an inflexion point, then $\dot{\kappa}\left(t^{*}\right)=-\frac{\rho\left(y\left(t^{*}\right)\right)}{y\left(t^{*}\right)^{2}} H=0$, and $H=0$ along the extremal. Let us now, consider $(17)$ for $\kappa \in]-1,1[$ (i.e. $\mathrm{u}=0$ ) and compute the second order time derivative of $\kappa$ :

$$
\ddot{\kappa}(t)=\frac{d}{d t}\left[-\frac{\rho(y)}{y^{2}} H+\kappa(t)\left(\alpha-\alpha y \frac{\rho^{\prime}(y)}{\rho(y)}\right)\right]=\frac{d}{d t}\left[\kappa(t)\left(\alpha-\alpha y \frac{\rho^{\prime}(y)}{\rho(y)}\right)\right]
$$

This computation shows that $\ddot{\kappa}\left(t^{\star}\right)=0$ but also that all the successive derivatives of $\kappa(t)$ are null at time $t^{\star}$. In fact, $\kappa\left(t^{\star}\right)$ is not an inflexion point and therefore $H>0, u(0)=1$ and $u\left(T_{f}\right)=-1$.

The rest of this section is divided into two parts. In the first one, the yield function $\rho(y)$ is assumed equal to some constant $0<\bar{\rho} \leq 1$. This assumption allows us to completely describe the solution to problem $\left(\mathcal{P}_{1}\right)$ with respect to the value of $T_{f}>T_{\min }$. In a second part, we build upon this basis and propose a methodology to search for the solution to the problem when the yield function is not constant.

\subsubsection{Constant yield function.}

First of all, let us remark that the solution to problem $\left(\mathcal{P}_{1}\right)$ doesn't depend on the value taken by $\bar{\rho}$. We therefore set $\bar{\rho}=1$. Up to now, we know the three following facts:

1. the control variable is equal to 1 over an interval of the form $[0, \varepsilon[$;

2. the control variable equals -1 over an interval of the form $\left[T_{f}-\varepsilon, T_{f}\right]$;

3. there might be singular arcs and such arcs are characterised by $\dot{y}=0$ with $y>0$.

An immediate consequence of the third fact is that there is no singular arc of the form $q=-y$ -i.e. with $u \in[-1,0]$. Indeed, $\dot{y}=0$ with $y>0$ can only be achieved with a strictly positive control. In the following, $\left.u_{\text {sing }} \in\right] 0,1[$ denotes a singular control. The following lemma states several facts allowing us to narrow down the list of candidate strategies. 


\section{Lemma 3.}

1. If the control variable switches to $u=0$ then it cannot be strictly positive again.

2. If the control variable switches to $u=-1$ then it cannot switch back to 0 .

3. There can be at most one singular arc.

4. For extremal trajectories comprising a singular arc:

(a) $y=\sqrt{\frac{q_{0}}{\alpha}}$ along the singular arc;

(b) the corresponding control is $u_{\text {sing }}=\sqrt{\alpha q_{0}}$;

(c) the following relation holds: $p=2 \sqrt{\alpha q_{0}}$.

Proof. 1. We use the quantity $\kappa(t)$. A commutation of the control from $u>0$ to $u=0$ means that, at some time $t^{\star}, \kappa\left(t^{\star}\right)=1$ and $\dot{\kappa}\left(t^{\star}\right)<0$. Let us write the expression of $\dot{\kappa}\left(t^{\star}\right)$ - cf. equation (17):

$$
\dot{\kappa}\left(t^{\star}\right)=\alpha \kappa\left(t^{\star}\right)-\frac{H}{y\left(t^{\star}\right)^{2}}<0
$$

Therefore, $\kappa(t)<\kappa\left(t^{\star}\right)$ over a small interval of the form $\left[t^{\star}, t^{\star}+\varepsilon[\right.$. Also, since the control switched to 0 , then the same goes for the variable $y(t)$. As a consequence, over $\left[t^{\star}, t^{\star}+\varepsilon[\right.$, we have:

$$
\dot{\kappa}(t)=\alpha \kappa(t)-\frac{H}{y(t)^{2}}<\dot{\kappa}\left(t^{\star}\right)<0
$$

As a consequence, $\kappa(t)$ remains less than 1 and the control cannot switch back to a positive value.

2. The second assertion is derived from similar arguments;

3. Assume that there exists an extremal trajectory that includes two singular arcs. Since this cannot happen as soon as $u=0$, then, the control sequence is of the following form - with $t_{4}<T_{f}$ :

$$
\begin{cases}u=1 & \text { for } t \in\left[0, t_{1}[\right. \\ u_{\text {sing }} & \text { for } t \in\left[t_{1}, t_{2}[\right. \\ u=1 & \text { for } t \in\left[t_{2}, t_{3}[\right. \\ u_{\text {sing }} & \text { for } t \in\left[t_{3}, t_{4}[\right.\end{cases}
$$

Along a singular arc, we have $\kappa=1$ and $\dot{\kappa}=0$, and equation (17) is rewritten:

$$
0=-\frac{H}{y(t)^{2}}+\alpha \Rightarrow y^{2}=\frac{H}{\alpha}=\frac{q_{0}}{\alpha}
$$

Since $y$ is constant along a singular arc, then $y^{2}\left(t_{1}\right)=\frac{q_{0}}{\alpha}$ and $y^{2}\left(t_{3}\right)=\frac{q_{0}}{\alpha}$. Those two equalities contradict the existence of two singular arcs. Indeed, $\dot{y} \neq 0$ over $\left[t_{2}, t_{3}[\right.$, therefore, $y\left(t_{1}\right)$ and $y\left(t_{3}\right)$ are not equal while having the same sign.

4. Items (a) and (b) come directly from the relation $y^{2}=\frac{q_{0}}{\alpha}$ used in the proof of fact (3). In order to prove item (c) we simply need to remark that, since both $\dot{\kappa}$ and $\dot{y}$ are null along a singular arc, the same is true for $\dot{q}$ :

$$
\begin{aligned}
0=\dot{q}=-\frac{\partial \mathcal{H}}{\partial y}(X, \mathcal{P}, u, 1) & =-p+\alpha q+|u| \\
& =-p+\alpha y+u_{\text {sing }} \\
& =-p+2 u_{\text {sing }}
\end{aligned}
$$


Remark 3. According to the informations gathered so far, control sequences candidate for optimality are:

1. $u=1$, then $u=0$ and finally $u=-1$;

2. $u=1$, then $u=u_{\text {sing }}, u=0$ and finally $u=-1$;

3. $u=1$, then $u=u_{\text {sing }}, u=1, u=0$ and finally $u=-1$.

Lemma 4. The following control sequence, with $\sqrt{\alpha q_{0}} \neq 1$, is not extremal

$$
u(t)=\left\{\begin{array}{cl}
1 & \text { for } t \in\left[0, t_{1}[\right. \\
\sqrt{\alpha q_{0}} & \text { for } t \in\left[t_{1}, t_{2}[\right. \\
1 & \text { for } t \in\left[t_{2}, t_{3}[\right. \\
0 & \text { for } t \in\left[t_{3}, t_{4}[\right. \\
-1 & \text { for } t \in\left[t_{4}, T_{f}\right]
\end{array}\right.
$$

Proof. Let us focus on the subinterval $\left[t_{2}, t_{3}\right.$ [ and show that time $t_{3}$ actually doesn't exist. Let $\bar{t}$ be the quantity $t-t_{2}$ and solve $\left(\Sigma_{1}\right)$ for $t>t_{2}$ with $u=1$ :

$$
y(t)=\left(y\left(t_{2}\right)-\frac{1}{\alpha}\right) e^{-\alpha \bar{t}}+\frac{1}{\alpha} \quad \text { and } \quad q(t)=\left(q\left(t_{2}\right)-\frac{p_{0}-1}{\alpha}\right) e^{\alpha \bar{t}}+\frac{p_{0}-1}{\alpha}
$$

The commutation of the control variable at time $t_{2}$ implies $y\left(t_{2}\right)=q\left(t_{2}\right)$, and the commutation at time $t_{3}$ implies $y\left(t_{3}\right)=q\left(t_{3}\right)$. Therefore, $t_{3}-t_{2}$ is the solution to the following equation:

$$
\left(y\left(t_{2}\right)-\frac{1}{\alpha}\right) e^{-\alpha \bar{t}}+\frac{1}{\alpha}=\left(q\left(t_{2}\right)-\frac{p_{0}-1}{\alpha}\right) e^{\alpha \bar{t}}+\frac{p_{0}-1}{\alpha}
$$

which is rewritten, denoting $q_{2}=q\left(t_{2}\right)$ :

$$
\left(q_{2}-\frac{1}{\alpha}\right) e^{-\alpha \bar{t}}+\frac{1}{\alpha}=\left(q_{2}-\frac{p_{0}-1}{\alpha}\right) e^{\alpha \bar{t}}+\frac{p_{0}-1}{\alpha}
$$

After a few simplifications, and denoting $X=e^{\alpha \bar{t}}$, we obtain:

$$
\left(\alpha q_{2}-p_{0}+1\right) X^{2}+\left(p_{0}-2\right) X-\alpha q_{2}+1=0
$$

We have, $y\left(t_{2}\right)=y\left(t_{1}\right)$, which implies $q_{2}=\sqrt{\frac{q_{0}}{\alpha}}$-cf. Lemma 3. Let us write the Hamiltonian at time $t=t_{2}$-i.e. $\mathcal{H}_{\mid t=t_{2}}=\mathcal{H}_{\mid t=0}=q_{0}$. We obtain the relations:

$$
q_{0}=\alpha q_{2}^{2} \quad \text { and } \quad q_{0}=p_{0} q_{2}-\alpha q_{2}^{2} \quad \underset{q_{2} \neq 0}{\Longrightarrow} \quad p_{0}=2 \alpha q_{2}
$$

Therefore, the equation given in (18) becomes:

$$
\left(1-\alpha q_{2}\right)(X-1)^{2}=0
$$

- If $\left(1-\alpha q_{2}\right)=0$, then $q_{0}=\alpha q_{2}^{2}=\frac{1}{\alpha}$, and $\sqrt{\alpha q_{0}}=1$ which is a contradiction.

- If $(X-1)=0$, then $X=1$ and $\bar{t}=0$, which means that $t_{3}>t_{2}$ doesn't exist.

As a consequence, the control strategy under consideration is not an extremal trajectory. 
Lemma 5. Consider the control sequence:

$$
u(t)=\left\{\begin{array}{cl}
1 & \text { for } t \in\left[0, t_{1}[\right. \\
\sqrt{\alpha q_{0}} & \text { for } t \in\left[t_{1}, t_{2}[\right. \\
0 & \text { for } t \in\left[t_{2}, t_{3}[\right. \\
-1 & \text { for } t \in\left[t_{3}, T_{f}\right]
\end{array}\right.
$$

1. If strategy (20) is extremal, then $t_{3}-t_{2}=\frac{1}{\alpha} \ln (1+\sqrt{2})$.

2. For given values of parameters $\alpha$ and $x_{f}$, there is a time $T_{\text {lim }}>0$ such that for all $T_{f}>$ $T_{\text {lim }}$, control sequence (20) is candidate for optimality.

Proof. 1. Using the notation $y_{1}=y\left(t_{1}\right)$, since the second arc is singular, we know that $y\left(t_{2}\right)=q\left(t_{2}\right)=y_{1}$. Let $\bar{t}$ be defined by $\bar{t}=t-t_{2}$, the expressions of $q$ and $y$ over the interval $\left[t_{2}, t_{3}\right]$ are:

$$
q(\bar{t})=y_{1} e^{\alpha \bar{t}}-\frac{p_{0}}{\alpha}\left(e^{\alpha \bar{t}}-1\right) \quad \text { et } \quad y(\bar{t})=y_{1} e^{-\alpha \bar{t}}
$$

At time $t_{3}$, the control commutes from 0 to -1 , which corresponds to the relation $q\left(t_{3}\right)=$ $-y\left(t_{3}\right)$. We now combine the relation $p_{0}=2 \sqrt{\alpha q_{0}}=2 u_{\text {sing }}$ from Lemma 3 , fact (4), with the fact that $y$ is constant and equals $y_{1}$ along the singular arc: $u_{\text {sing }}=\alpha y_{1}$ and $p_{0}=2 \alpha y_{1}$.

Let us now compute $\bar{t}$ such that $-y(\bar{t})=q(\bar{t})$ :

$$
\begin{array}{rlr}
-y_{1} e^{-\alpha \bar{t}} & =y_{1} e^{\alpha \bar{t}}-\frac{p_{0}}{\alpha}\left(e^{\alpha \bar{t}}-1\right) & \\
-y_{1} e^{-\alpha \bar{t}} & =y_{1} e^{\alpha \bar{t}}-\frac{2 \alpha y_{1}}{\alpha}\left(e^{\alpha \bar{t}}-1\right) & \\
-e^{-\alpha \bar{t}} & =e^{\alpha \bar{t}}-2\left(e^{\alpha \bar{t}}-1\right) & \\
-1 & =e^{2 \alpha \bar{t}}-2\left(e^{2 \alpha \bar{t}}-e^{\alpha \bar{t}}\right) \\
0 & =X^{2}-2 X-1 \quad \quad\left(\text { since } y_{1}>0\right) \\
&
\end{array}
$$

Therefore $X=1 \pm \sqrt{2}$, which yields $t_{3}-t_{2}=\frac{1}{\alpha} \ln (1+\sqrt{2})$.

2. Consider the following procedure.

- Let $t_{1}=t_{2}$.

- Let $t_{3}-t_{2}=\frac{1}{\alpha} \ln (1+\sqrt{2})$, set $T_{f}-t_{3}=T_{f}-\frac{1}{\alpha} \ln (1+\sqrt{2})-t_{1}$ and solve $y\left(T_{f}\right)=0$ for $t_{1}$. It is the following function of $\alpha$ and $T_{f}$ :

$$
t_{1}=\frac{1}{\alpha} \ln \left[\left(1-\frac{\sqrt{2}}{2}\right)\left(1+e^{\alpha T f}\right)\right]
$$

- For a given final time $T_{f}$, control strategy (20) leads to:

$$
x_{f}=-\frac{T f}{\alpha}+\frac{\ln (1+\sqrt{2})}{\alpha^{2}}+\frac{2}{\alpha^{2}} \ln \left(\left(1-\frac{\sqrt{2}}{2}\right)\left(1+e^{\alpha T f}\right)\right)
$$


- The above relation is reversed in order to express $T_{f}$, as a function of $\alpha$ and $x_{f}$. We obtain the following relations, and $T_{l i m}$ is the latter one:

$$
\begin{aligned}
& \frac{1}{\alpha} \ln \left((1+\sqrt{2}) e^{\alpha^{2} x_{f}}-1-\sqrt{\left((1+\sqrt{2}) e^{\alpha^{2} x_{f}}-1\right)^{2}-1}\right) \leq 0 \\
& \text { and } \\
& \frac{1}{\alpha} \ln \left((1+\sqrt{2}) e^{\alpha^{2} x_{f}}-1+\sqrt{\left((1+\sqrt{2}) e^{\alpha^{2} x_{f}}-1\right)^{2}-1}\right)>0
\end{aligned}
$$

The only strategy left to investigate is the following one:

$$
u(t)=\left\{\begin{array}{cl}
1 & \text { for } t \in\left[0, t_{1}[\right. \\
0 & \text { for } t \in\left[t_{1}, t_{2}[\right. \\
-1 & \text { for } t \in\left[t_{2}, T_{f}\right]
\end{array}\right.
$$

For given values of $\alpha, x_{f}, T_{f}>T_{\min }$, there always exists a control of the form (21) joining $(0,0)^{t}$ to $\left(x_{f}, 0\right)^{t}$. From the two constraints $y\left(T_{f}\right)=0$ and $x\left(T_{f}\right)=x_{f}$, we compute the expressions of $t_{1}$ et $t_{2}$ :

$$
\left\{\begin{array}{l}
t_{1}=\frac{1}{\alpha} \ln \left[\frac{1}{2}\left(1+e^{\alpha T_{f}}-\sqrt{1+2 e^{\alpha T_{f}}+e^{2 \alpha T_{f}}-4 e^{\alpha^{2} x_{f}+\alpha T_{f}}}\right)\right] \\
t_{2}=\frac{1}{\alpha} \ln \left[\left(1-e^{\alpha t_{1}}+e^{\alpha T_{f}}\right)\right]
\end{array}\right.
$$

The trajectory generated by control (21) is clearly optimal when $T_{\min }<T_{f}<T_{l i m}$. Indeed, there is no other extremal trajectory.

Theorem 2 (Optimal strategy for a constant yield function).

For a given set of parameters $\alpha, x_{f}$ and $T_{f}$, let $X(t): t \mapsto(x(t), y(t))^{t}$ denote the trajectory of $\left(\Sigma_{1}\right)$ steering $(0,0)^{t}$ to $\left(x_{f}, 0\right)^{t}$ under a control sequence of the form (21).

1. If $\left(X(t), p_{0}, q(t)\right)$ is an extremal trajectory of Pontryagin's maximum principle, then $p_{0}$ and $q_{0}$ are given by the following expressions:

$$
\begin{aligned}
& p_{0}=\frac{e^{-\alpha t_{2}}\left(e^{\alpha t_{1}}-1\right)\left(1+e^{2 \alpha\left(t_{2}-t_{1}\right)}\right)}{e^{\alpha\left(t_{2}-t_{1}\right)}-1} \\
& q_{0}=\frac{e^{-2 \alpha t_{1}}}{\alpha}\left(e^{\alpha t_{1}}-1\right)\left(1+e^{\alpha t_{1}}(p o-1)\right)
\end{aligned}
$$

2. For $T_{f}>T_{\text {lim }}$, with $T_{\text {lim }}$ as in Lemma 5, then $\left(X(t), p_{0}, q(t)\right)$ is not an extremal trajectory.

3. The solution to the optimal control problem $\left(\mathcal{P}_{1}\right)$ on a flat road, with drag coefficient $\alpha$ and constant yield function is:

(a) for $T_{\min }<T_{f} \leq T_{\text {lim }}$, with $T_{\text {lim }}$ as in Lemma 5, the optimal control strategy is (21);

(b) for $T_{f}>T_{\text {lim }}$, the optimal control strategy is (20).

Proof. 1. Parameters $\alpha, x_{f}, T_{f}$ being fixed, the commutation times $t_{1}$ et $t_{2}$ of control sequence (21) are given by (22), which allows the computation of $y\left(t_{1}\right)$ and $y\left(t_{2}\right)$.

(a) $p_{0}$ is determined with the help of the expression of $q(t)$ over the interval $\left[t_{1}, t_{2}\right]$ :

$$
q(t)=q\left(t_{1}\right) e^{\alpha\left(t-t_{1}\right)}-\frac{p_{0}}{\alpha}\left(e^{\alpha\left(t-t_{1}\right)}-1\right)
$$

Since $q\left(t_{1}\right)=y\left(t_{1}\right)$, the equation $q\left(t_{2}\right)=-y\left(t_{2}\right)$ is solved for $p_{0}$. 
(b) $q_{0}$ is determined from the expression of $q(t)$ over the interval $\left[0, t_{1}\right]$ :

$$
q(t)=q_{0} e^{\alpha t}+\frac{1}{\alpha}\left(1-p_{0}\right)\left(e^{\alpha t}-1\right) .
$$

In both cases, the derived expression is uniquely determined.

2. For a given set of parameters $\alpha, x_{f}, T_{f}$, assume that $\left(X(t), p_{0}, q(t)\right)$ is an extremal trajectory. Then, $t_{1}, p_{0}$ et $q_{0}$ can be explicitly computed. Therefore, we can determine the expressions of both $y(t)$ and $q(t)$ over the interval $\left[0, t_{1}\right]$. A commutation takes place if there is a time $t$ such that $y(t)=q(t)$. This equation is then solved for $t$.

We obtain two expressions ${ }^{5}$. One of them is exactly formula $t_{1}$ given in (22). However, the other one is real and lower than $t_{1}$ as soon as $T_{f}>T_{l i m}$. In other words, for $T_{f}>T_{\text {lim }}$ a first commutation of the control happens before time $t_{1}$ and consequently the control sequence (21) is not extremal.

3. The analysis performed in the present section demonstrated that there are only two possible extremal strategies. They have the following restrictions:

- the trajectory due to the control sequence (21) is not extremal as soon as $T_{f}>T_{\text {lim }}$;

- the trajectory due to the control sequence (20) doesn't exist for $T_{\min }<T_{f} \leq T_{\text {lim }}$.

Figure 8 illustrates Theorem 2 for $x_{f}=6$ and $\alpha=0.5$. The quantity $T_{\text {lim }}$ is determined as explained in Lemma 5. Figures $8 \mathrm{a}$ and $8 \mathrm{~b}$ show the optimal trajectory for $T_{f}<T_{\text {lim }}$, and Figures $8 \mathrm{c}$ and $8 \mathrm{~d}$ correspond to the case $T_{f}>T_{\text {lim }}$. In particular, Figures $8 \mathrm{e}$ and $8 \mathrm{f}$ illustrate the fact that strategy (21) is not extremal for $T_{f}>T_{l i m}$. Those last two figures are obtained as follows: $t_{1}$ and $t_{2}$ are determined in order to steer $X(0)$ to $X\left(T_{f}\right)$ using strategy (21). When we apply this control to the Hamiltonian system, we see that trajectory $q(t)$ intersects $y(t)$ at some time $0<t<t_{1}$, showing as expected that this trajectory is not extremal.

\subsubsection{Non-constant yield function.}

In this section, in order to address $\left(\mathcal{P}_{1}\right)$ on a flat road, with a drag coefficient $\alpha>0$ and a non-constant yield function $\rho(y)$, we propose the following methodology based on fact (3) of Theorem 2.

1. Compute the trajectory of $\left(\Sigma_{1}\right)$ given by control sequence (21).

2. If possible, compute the trajectory of $\left(\Sigma_{1}\right)$ given by control sequence (20).

3. Check which one of those two trajectories is extremal, and compute the cost explicitly to remove possible ambiguities.

4. It can happen that neither strategy (21) nor strategy (20) yield extremal trajectories. Indeed, the facts of Lemma 3 don't hold anymore and there can be more than one commutation of the control variable between the values $u=0$ and $u=-1$. When this situation arises, a new control strategy is investigated —see (25) below.

A trajectory of $\left(\Sigma_{1}\right)$, given by the control sequence (21), such that $X\left(T_{f}\right)=\left(x_{f}, 0\right)^{t}$ is still given by relations (22). However, for a trajectory given by control sequence (20), relation $t_{3}-t_{2}=\frac{1}{\alpha} \ln (1+\sqrt{2})$ of Lemma 5 doesn't hold anymore, we have to rely on a numerical scheme.

\footnotetext{
${ }^{5}$ Since they are quite large, those two expressions are not reproduced here. However, the interested reader can obtain them with the help of a symbolic computation software, or by consulting [?].
} 


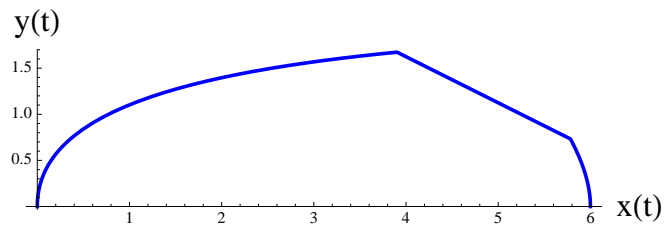

(a)

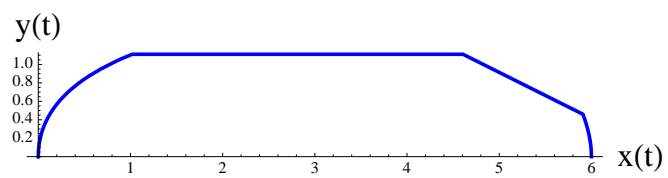

(c)

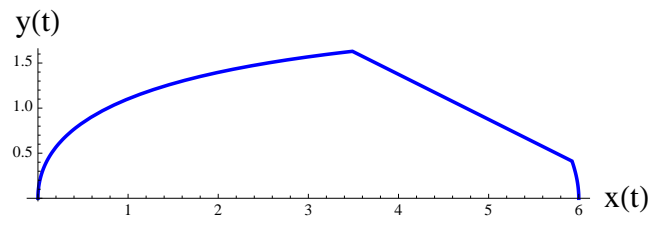

(e)

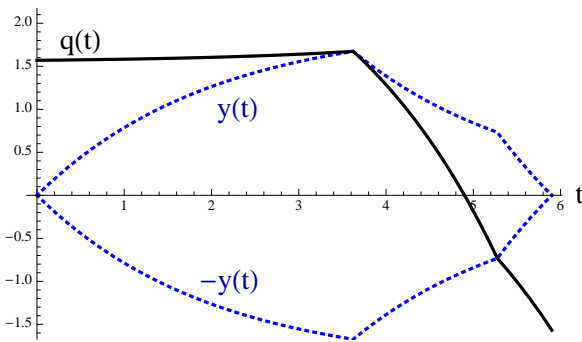

(b)

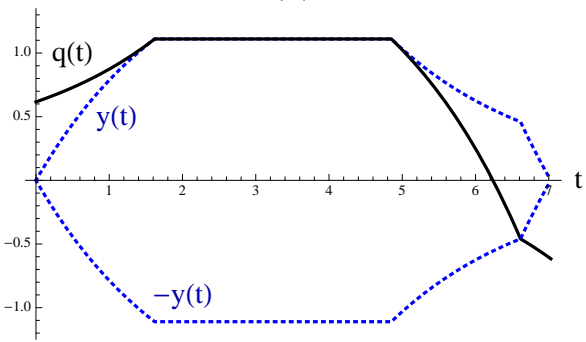

(d)

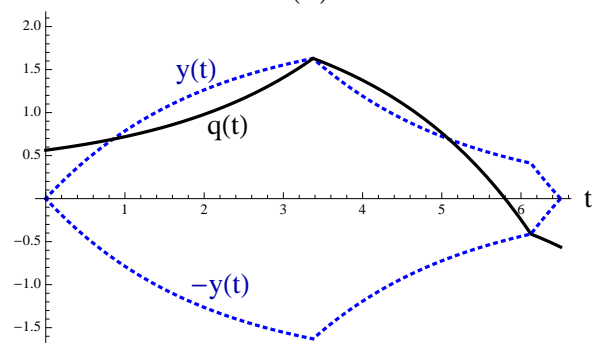

(f)

Figure 8: Optimal and non-optimal trajectories for the second case study with a constant yield function. Parameters: $\alpha=0.5, x_{f}=6, T_{\min } \approx 5.52$ and $T_{l i m} \approx 5.95$.

Figures $8 \mathrm{a}$ and $8 \mathrm{~b}$ correspond to $T_{f}=5.9$. Figures $8 \mathrm{c}$ to $8 \mathrm{f}$ correspond to $T_{f}=6.5$.

\section{Computing extremal trajectories given by strategy (20).}

First, we need to derive usable expressions for $p_{0}$ and $q_{0}$. Along a singular arc, since $\dot{y}=0$, then $\dot{q}=\frac{d}{d t}\left(\frac{y}{\rho(y)}\right)=0$. Let $y_{s}$ denote the value of $y(t)$ along the singular arc, then $u_{s i n g}=\alpha y_{s}$ and:

$$
\begin{aligned}
& 0=\dot{q}=-\frac{\partial}{\partial y} \mathcal{H}=\frac{\left|u_{\text {sing }}\right|}{\rho\left(y_{s}\right)}-\frac{y_{s}\left|u_{\text {sing }}\right| \rho^{\prime}\left(y_{s}\right)}{\rho^{2}\left(y_{s}\right)}+\alpha q_{s}-p_{0} \quad \text { where } \quad q_{s}=\frac{y_{s}}{\rho\left(y_{s}\right)} \text { and } \rho^{\prime}(y)=\frac{\partial \rho}{\partial y}(y) \\
& \Rightarrow p_{0}=\frac{2 \alpha y_{s}}{\rho\left(y_{s}\right)}-\frac{\alpha y_{s}^{2} \rho^{\prime}\left(y_{s}\right)}{\rho^{2}\left(y_{s}\right)}
\end{aligned}
$$

Now, since $u_{0}=1$, and denoting by $\mathcal{H}_{s}$ the Hamiltonian function computed along a singular arc, we have:

$$
\mathcal{H}_{\mid t=0}=q_{0}=\mathcal{H}_{s}=p_{0} y_{s}+q_{0}\left(u_{\text {sing }}-y_{s}\right)-\frac{y_{s}\left|u_{\text {sing }}\right|}{\rho\left(y_{s}\right)} \Rightarrow q_{0}=p_{0} y_{s}-\frac{\alpha y_{s}^{2}}{\rho\left(y_{s}\right)}
$$


In order to compute an extremal trajectory associated to (20), the following methodology is proposed:

1. set $t_{1}$ to some arbitrary value, then $y\left(t_{2}\right)=y\left(t_{1}\right)=y_{s}$ and both $p_{0}$ and $q_{0}$ can be computed;

2. the duration $\left(t_{3}-t_{2}\right)$ is the solution to $q(t)=-\frac{y(t)}{\rho(y(t))}$ where $q(t)$ and $y(t)$ are the trajectories of the Hamiltonian system associated to $\left(\mathcal{P}_{1}\right)$ with $u=0, y(0)=y_{s}$ and $q(0)=\frac{y_{s}}{\rho\left(y_{s}\right)}$;

3. the duration $\left(T_{f}-t_{3}\right)$ comes from the constraint $y(t)=0$ where $y(t)$ is the trajectory of $\left(\Sigma_{1}\right)$ with $u=-1$ and setting $y(0)$ to the value taken by $y$ in the previous step when we solved equation $q(t)=-\frac{y(t)}{\rho(y(t))}$;

4. $t_{1}$ is determined in order to solve the equation $x\left(T_{f}\right)=x_{f}$, with the duration of the singular arc (i.e. $t_{2}-t_{1}$ ) given by $T_{f}$ minus the length of the three other arcs.

Remark 4. - Each step of this methodology is achieved with a simple dichotomy.

- For a given set of parameters $\alpha$ and $x_{f}$, the minimum time $T_{l i m}$ for the existence of a singular strategy (20) is determined with a similar methodology: the singular arc is reduced to a point but the constraints are kept. Time $t_{1}$ is determined such that $x\left(T_{f}\right)=x_{f}$, which yields the value of $T_{\text {lim }}=T_{f}$.

- In practice, if a singular strategy exists, then the basic strategy (21) is not extremal.

\section{Trajectories having a two-step braking behaviour.}

The two control strategies presented so far - i.e. (20) and (21) - are not sufficient to account for all the situations we encountered during the resolution of this case. Since Lemma 3 doesn't guarantee anymore that each type of commutation happens at most once, we considered a strategy of the form (25), which is detailed below.

Let us remember that the running cost is $\frac{|y u|}{\rho(y)}$. On the one hand, the fact that it tends to zero when $y$ tends to 0 points out the interest of braking at low speed. On the other hand, a non-null control is expected to take place when the yield function's value is the largest. Actually, strategies (20) and (21) both realise a tradeoff between those two behaviours, and the fact that both strategies aren't extremal hints toward a two-step braking strategy. In other words, $u=-1$ a first time when the yield function is large enough, and a second time when the speed is low enough.

Actually, the following strategy produces extremal trajectories when (20) and (21) fail to do so. Those trajectories are calculated with a methodology adapted from the one used for strategy (20).

$$
u(t)=\left\{\begin{array}{cll}
1 & \text { when } & t \in\left[0, t_{1}[\right. \\
\alpha y_{1} & \text { when } t \in\left[t_{1}, t_{2}[\right. \\
0 & \text { when } t \in\left[t_{2}, t_{3}[\right. \\
-1 & \text { when } t \in\left[t_{3}, t_{4}[\right. \\
0 & \text { when } t \in\left[t_{4}, t_{5}[\right. \\
-1 & \text { when } t \in\left[t_{5}, T_{f}\right]
\end{array}\right.
$$

Figures 9, 10 and 11 illustrate the optimal trajectories calculated following the above methodology for fixed parameters $x_{f}=6$ and $\alpha=0.5$. Figure 9 corresponds to the case $T_{\min }<T_{f}<$ $T_{\text {lim }}$, and Figures 10 and 11 to $T_{f}>T_{\text {lim }}$. For $T_{f}=10$, neither (21) nor (20) produce extremal trajectories, but the new strategy (25) does. It is illustrated in Figure 11. In fact, this situation 


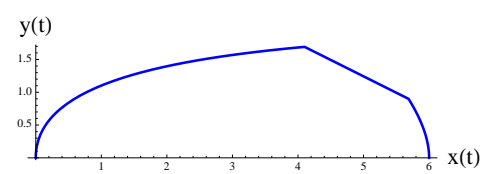

(a)

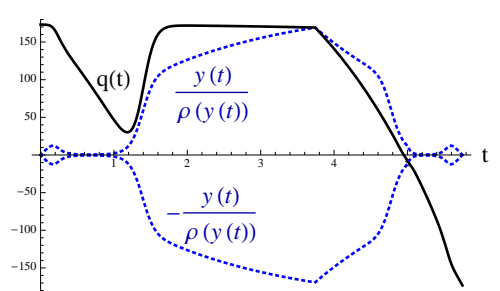

(b)

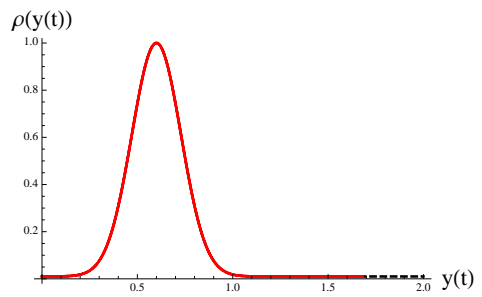

(c)

Figure 9: Optimal trajectory for the second case study with a non-constant yield function. Parameters: $\alpha=0.5, x_{f}=6, T_{\min } \approx 5.52, T_{\text {lim }} \approx 5.8$ and $T_{f}=5.75$.

happens only for $T_{f}$ within a rather small interval of time. In Figures $9 \mathrm{c}, 10 \mathrm{c}$ and $11 \mathrm{f}$ the visited values of the yield function are displayed in red. The blue sections in Figure 11f correspond to the two braking periods. Figure 11c shows the trajectory of the adjoint variable $q(t)$ while zooms on at start and final times are shown in Figures 11d and 11e.

Remark 5. Extremal trajectories such as the one displayed in Figure 11 don't exist for all type of non-constant yield function. Indeed, in the case of the yield function shown in Figure \%c, where $\rho(0)=0.2$, there is no such extremal. This can be understood as a translation of the fact that, since the ratio between the maximum and the minimum of the yield is not significant, it is more interesting to brake at low speed only.

\subsection{Non-flat road}

In this third case study, we consider a non-flat road and no drag coefficient. System $\left(\Sigma_{1}\right)$, and the behaviour of the road profile under consideration (represented in Figure 12) are:

$$
\left(\Sigma_{1}\right)\left\{\begin{array} { l } 
{ \dot { x } = y } \\
{ \dot { y } = u - \operatorname { s i n } ( \theta ( x ) ) }
\end{array} \quad \text { where } \quad \left\{\begin{array}{l}
\theta(x) \text { is decreasing for } x \in[-10,-6.5[ \\
\theta(x) \text { is increasing for } x \in]-6.5,-1[ \\
\theta(x) \text { is constant for } x \geq-1
\end{array}\right.\right.
$$

In order to simplify notations when there is no ambiguity, we denote $K=\sin (\theta(x))$ for $x \geq-1$. The minimum time is $T_{\min }=\frac{2 \sqrt{x_{f}}}{\sqrt{1-K^{2}}}$, and we set $x_{f}=2$.

As before, abnormal extremals are out of the picture. First, let $T_{f}^{1,0}$ be the time needed in order to reach $X=\left(x_{f}, 0\right)^{t}$ with the strategy

$$
u(t)= \begin{cases}1 & \text { for } t \in\left[0, t_{c}[\right. \\ 0 & \text { for } t \in\left[t_{c}, T_{f}^{1,0}\right]\end{cases}
$$

Lemma 6. 1. If the control sequence (26) is extremal, then the Hamiltonian $H$ is null along the corresponding trajectory of $\left(\Sigma_{1}\right)$;

2. we have the following equivalence:

$$
\rho^{\prime}\left(y\left(t_{c}\right)\right) \geq 0 \quad \Longleftrightarrow \quad \text { (26) is an extremal strategy }
$$




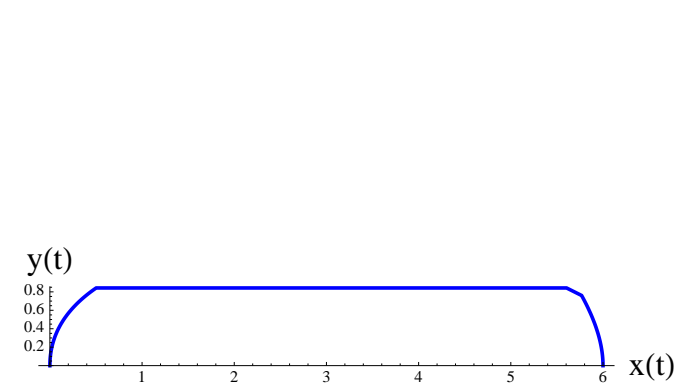

(a)

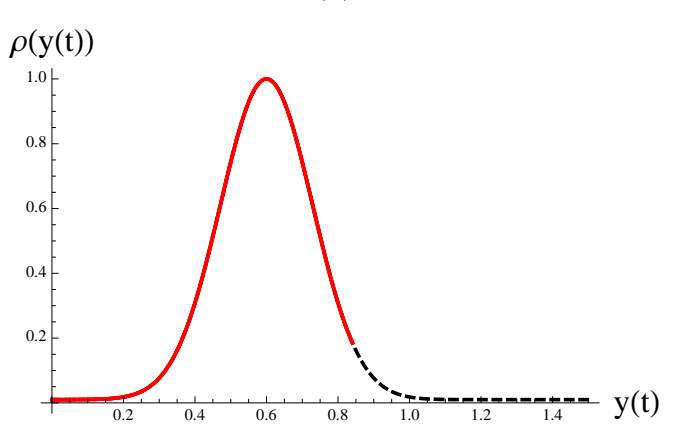

(c)

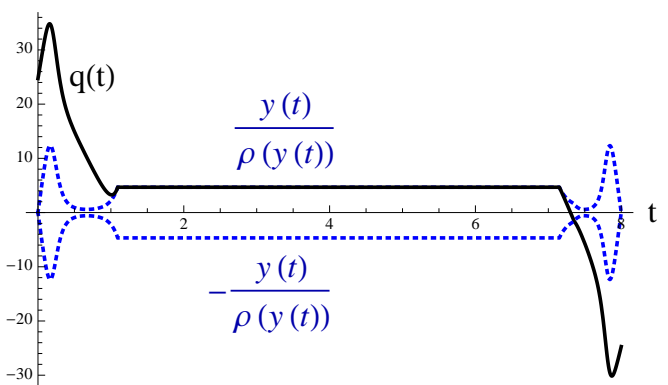

(b)

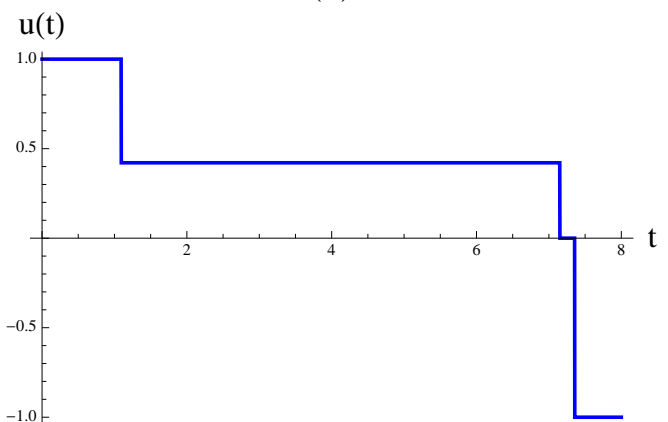

(d)

Figure 10: Optimal trajectory for the second case study with a non-constant yield function. Parameters: $\alpha=0.5, x_{f}=6, T_{\min } \approx 5.52, T_{l i m} \approx 5.8$ and $T_{f}=8$. 
3. for all $T_{f}>T_{f}^{1,0}$ the following strategy is also extremal:

$$
u(t)=\left\{\begin{array}{cl}
K & \text { for } t \in\left[0, t_{1}[\right. \\
1 & \text { for } t \in\left[t_{1}, t_{1}+t_{c}[\right. \\
0 & \text { for } t \in\left[t_{1}+t_{c}, T_{f}-t_{2}[\right. \\
K & \text { for } t \in\left[T_{f}-t_{2}, T_{f}[\right.
\end{array}\right.
$$

where $t_{1} \geq 0, t_{2} \geq 0$ and $t_{1}+t_{2}=T_{f}-T_{f}^{1,0}$.

Proof. 1. The normal Hamiltonian, with $x \geq-1$, is $\mathcal{H}(X, \mathcal{P}, u, 1)=-\frac{|y u|}{\rho(y)}+p y+q(u-K)$.

For an extremal trajectory given by $(26), u\left(T_{f}\right)=0$ and $\frac{y\left(T_{f}\right)}{\rho\left(y\left(T_{f}\right)\right)} \leq q\left(T_{f}\right) \leq-\frac{y\left(T_{f}\right)}{\rho\left(y\left(T_{f}\right)\right)}$. Since $y\left(T_{f}\right)=0$ then $q\left(T_{f}\right)=0$, and the Hamiltonian expressed at time $T_{f}$ yields $H=0$.

2. Let us assume $\rho^{\prime}\left(y_{c}\right) \geq 0$, with $y_{c}=y\left(t_{c}\right)$. Since $u=1$ over $\left[0, T_{c}[\right.$, then $y \geq 0$ over ] $\left.0, t_{c}\right]$. Let $p=p_{0}$ be a constant, and $q(t)$ be the solution to

$$
\dot{q}=-\frac{\partial}{\partial y}\left(-\frac{y}{\rho(y)}+p y+q(1-K)\right) \quad \text { with } q(0)=0
$$

We compute the time derivative of $\mathcal{H}(X, \mathcal{P}, u, 1)$ over $] 0, t_{c}[$. Recall that $y>0$ and $u$ is constant, which yields $\dot{\mathcal{H}}=0$. Therefore, $\mathcal{H}$ is constant over $\left[0, t_{c}\right]$ by continuity. Now, since we've set $q_{0}=0$, then $\mathcal{H}_{\mid t=0}=0$, and therefore $\mathcal{H}_{\mid t=t_{c}}=0$.

The commutation at time $t_{c}$ happens if $q\left(t_{c}\right)=\frac{y_{c}}{\rho\left(y_{c}\right)}$, which is true if $p_{0}=\frac{K}{\rho\left(y_{c}\right)}$. In order to show that (26) is an extremal strategy, we still have to show that:

(a) $q(t)>\frac{y}{\rho(y)}$ over $] 0, t_{c}[$;

(b) $-\frac{y}{\rho(y)}<q(t)<\frac{y}{\rho(y)}$ over $] t_{c}, T_{f}^{1,0}[$.

- Over $] 0, t_{c}[$ :

$$
-\frac{y}{\rho(y)}+p_{0} y+q(1-K)=0 \quad \Longleftrightarrow \quad q=\frac{y}{1-K}\left(\frac{1}{\rho(y)}-p_{0}\right)
$$

After a few manipulations, and replacing $p_{0}$ by $\frac{K}{\rho\left(y_{c}\right)}$, we obtain the expression:

$$
q-\frac{y}{\rho(y)}=\frac{K}{1-K} y\left(\frac{1}{\rho(y)}-\frac{1}{\rho\left(y_{c}\right)}\right)
$$

Since $\rho^{\prime}\left(y_{c}\right) \geq 0$ and $0<y(t)<y_{c}$ over $] 0, t_{c}\left[\right.$, then $\left(\frac{1}{\rho(y)}-\frac{1}{\rho\left(y_{c}\right)}\right)>0$ and $q>\frac{y}{\rho(y)}$.

- Over $] t_{c}, T_{f}^{1,0}[$ :

we have $u=0$. Let $\bar{t}=t-t_{c}$, then $y(\bar{t})=-K \bar{t}+y_{c}$ and $q(\bar{t})=-p_{0} \bar{t}+q\left(t_{c}\right)=$ $-\frac{K}{\rho\left(y_{c}\right)} \bar{t}+\frac{y_{c}}{\rho\left(y_{c}\right)}$. Hence $T_{f}^{1,0}-t_{c}=\frac{y_{c}}{K}, q\left(T_{f}^{1,0}\right)=0$ and trivially $-\frac{y}{\rho(y)}<q$ over ]$t_{c}, T_{f}^{1,0}[$.

Let us now determine the sign of $q-\frac{y}{\rho(y)}$ : 


$$
\begin{aligned}
q-\frac{y}{\rho(y)} & =\frac{y_{c}}{\rho\left(y_{c}\right)}-\frac{K}{\rho\left(y_{c}\right)} \bar{t}-\frac{y}{\rho(y)} \\
& =\frac{y_{c}}{\rho\left(y_{c}\right)}-\frac{K}{\rho\left(y_{c}\right)} \bar{t}-\frac{y_{c}-K \bar{t}}{\rho(y)} \\
& <\frac{y_{c}}{\rho\left(y_{c}\right)}-\frac{K}{\rho\left(y_{c}\right)} \bar{t}-\frac{y_{c}-K \bar{t}}{\rho\left(y_{c}\right)}=0 \quad\left(\text { since } y<y_{c}\right) \text { and }\left(y_{c}-K \bar{t}>0\right)
\end{aligned}
$$

This proves the result, and strategy (26) is extremal.

Let us assume $\rho^{\prime}\left(y_{c}\right)<0$, with $y_{c}=y\left(t_{c}\right)$. As we've done before, we consider the quantity $\kappa(t)=\frac{q \rho(y)}{y}$ over an interval of time that contains $t_{c}$ and such that $y>0$. We assume that (26) is an extremal strategy and compute the time derivative of $\kappa(t)$ over this interval:

$$
\dot{\kappa}(t)=-\frac{H \rho(y)}{y^{2}}-\frac{|u| \rho^{\prime}(y)}{\rho(y)}+\kappa(t) \frac{\rho^{\prime}(y)}{\rho(y)} \dot{y}
$$

When $t=t_{c}, \kappa\left(t_{c}\right)=1$ and $u \geq 0$, which yields $\dot{\kappa}\left(t_{c}\right)=-\frac{\rho^{\prime}\left(y\left(t_{c}\right)\right)}{\rho\left(y\left(t_{c}\right)\right)} K$ since $H=0$. As a consequence, a commutation of the control cannot take place since $\dot{\kappa}\left(t_{c}\right)>0$. This contradicts the fact that (26) is extremal.

3. This item comes from the two following facts.

(a) An extremal trajectory having a singular arc of the form $y=0, \dot{y}=0$ implies $H=0$.

(b) If (26) is an extremal strategy, then $H=0$.

We are now left with the following question: what happens when $\rho^{\prime}\left(y_{c}\right)<0$ ? We know from Lemma 6 that this strategy cannot be extremal. From the experience gained with the preceding case study, searching for singular arcs comes to mind. As we have seen at the beginning of Section 4: since $\theta(x)$ is constant, then singular arcs are of the form $\dot{y}=0$. A possible strategy is:

$$
u(t)= \begin{cases}1 & \text { for } t \in\left[0, t_{c}[\right. \\ K & \text { for } t \in\left[t_{c}, t_{c}+t_{\text {sing }}[\right. \\ 0 & \text { for } t \in\left[t_{c}+t_{\text {sing }}, T_{f}^{1, s, 0}\right]\end{cases}
$$

The associated trajectory of $\left(\Sigma_{1}\right)$ has a singular arc over the time interval $\left[t_{c}, t_{c}+t_{\text {sing }}[\right.$ which can be as large as needed in order to meet the constraint $x\left(T_{f}\right)=x_{f}$. In fact, if we assume that strategy (29) is extremal, we have the following facts:

1. $H=0$ since $u(t)=0$ for $t \in\left[t_{c}+t_{\text {sing }}, T_{f}\right]$;

2. the time derivative of the quantity $\kappa=\frac{q \rho(y)}{y}$, computed at time $t_{c}$ is $\dot{\kappa}\left(t_{c}\right)=-\frac{\rho^{\prime}\left(y\left(t_{c}\right)\right)}{\rho\left(y\left(t_{c}\right)\right)} K$ - see formula (28) - which shows that a singular arc can happen only if $\rho^{\prime}\left(y\left(t_{c}\right)\right)=0$.

The final time $T_{f}^{1, s, 0}$ is uniquely determined by the following procedure, and the corresponding trajectory is proven extremal by using the same arguments as in Lemma 6:

- $t_{c}$ is such that $\rho($.$) reaches its maximum over \mathbb{R}^{+, \star}$ at $y\left(t_{c}\right)$; 
- $t_{\text {sing }}$ and $T_{f}^{1, s, 0}$ are such that $X\left(T_{f}^{1, s, 0}\right)=\left(x_{f}, 0\right)^{t}$.

As before, if (29) is an extremal strategy, then for all $T_{f}>T_{f}^{1, s, 0}$ the following strategy is also extremal:

$$
u(t)=\left\{\begin{array}{cl}
K & \text { for } t \in\left[0, t_{1}[\right. \\
1 & \text { for } t \in\left[t_{1}, t_{1}+t_{c}[\right. \\
K & \text { for } t \in\left[t_{1}+t_{c}, t_{1}+t_{c}+t_{\text {sing }}[\right. \\
0 & \text { for } t \in\left[t_{1}+t_{c}+t_{\text {sing }}, T_{f}-t_{2}[\right. \\
K & \text { for } t \in\left[T_{f}-t_{2}, T_{f}\right]
\end{array}\right.
$$

where $t_{1} \geq 0, t_{2} \geq 0$ and $t_{1}+t_{2}=T_{f}-T_{f}^{1, s, 0}$. This strategy is illustrated in Figure 13 .

Let us now define $T_{b a c k}$ as follows:

$$
T_{\text {back }}=\max _{\mathbb{R}^{+*}}\left\{T_{f} \text { such that } \forall u \in L^{\infty}\left[0, T_{f}\right]\left\{\begin{aligned}
X(0) & =X_{0}, X\left(T_{f}\right)=X_{f} \\
\theta(x(t)) & =\theta(0)
\end{aligned} \quad \forall t \in\left[0, T_{f}\right]\right\}\right.
$$

We call small time trajectory a trajectory having a final time $T_{f}$ within the interval $\left.] T_{\min }, T_{\text {small }}\right]$, where $T_{\text {small }}=\min \left(T_{\text {back }}, T_{f}^{1,0}\right)$.

Lemma 7 (Small time trajectories). 1. An optimal small time trajectory of $\left(\mathcal{P}_{1}\right)$ has no backward arc.

2. Small time extremals of $\left(\mathcal{P}_{1}\right)$ do not contain singular arcs of the form $y=0$.

3. Let $y_{s}$ be the constant speed of the singular arc of a small time extremal, then $\frac{\partial \rho}{\partial y}\left(y_{s}\right)<0$.

4. Consider a small time extremal trajectory containing a singular arc, and denote $y_{s}$ the constant speed, then:

$$
q_{0}=\frac{K y_{s}^{2} \rho^{\prime}\left(y_{s}\right)}{(K-1) \rho\left(y_{s}\right)^{2}} \quad \text { and } \quad p_{0}=K \frac{\rho\left(y_{s}\right)-y_{s} \rho^{\prime}\left(y_{s}\right)}{\rho\left(y_{s}\right)^{2}}
$$

Proof. 1. Since $T_{f}<T_{\text {back }}$, trajectories $(x(t), y(t))^{t}$ of $\left(\mathcal{P}_{1}\right)$ starting from $(0,0)^{t}$ can reach $\left(x_{f}, 0\right)^{t}$ only by staying on portions of road such that $\theta(x(t)) \equiv$ constant. Then, $\left(\mathcal{P}_{1}\right)$ can be viewed as invariant with respect to translations along the $x$-axis. According to Lemma 2, an optimal trajectory has no backward arc.

2. In the problem under consideration, $T_{b a c k}<T_{f}^{1,0}$. As a consequence, for a small time extremal, $y\left(T_{f}\right)=0$ cannot be achieved with a strategy of the form (26), and $u$ must be negative over some time interval. Hence, there exists a non-trivial interval of the form $] \tilde{t}, \bar{t}[$ such that:

- $y>0$ for all $t \in] \tilde{t}, \bar{t}\left[\right.$ and the the quantity $\kappa(t)=\frac{q \rho(y)}{y}$ exists for all $\left.t \in\right] \tilde{t}, \bar{t}[$;

- $\kappa(\tilde{t})=1$ and $\kappa(\bar{t})=-1$

- there is a $\left.t^{\star} \in\right] \tilde{t}, \bar{t}\left[\right.$ such that $\kappa\left(t^{\star}\right)=0$ and $\dot{\kappa}\left(t^{\star}\right)<0$.

The time derivative of $\kappa(t)$, over this interval is, again, equation (28). When $\kappa(t)=0$, then $u=0$ and $\dot{\kappa}\left(t^{\star}\right)=-\frac{H \rho(y)}{y^{2}}$ which implies that $H$ must be strictly positive in order to achieve $\dot{\kappa}\left(t^{\star}\right)<0$. A singular arc with $y=0$ cannot exist since it implies $H=0$. 
3. As before, we define the quantity $\kappa(t)=\frac{q \rho(y)}{y}$ over intervals where $y \neq 0$, and we derive an expression for $\dot{\kappa}(t)$ - see equation (28) above. Along a singular arc, we have $\kappa(t)=1$ and $\dot{\kappa}(t)=0$, following expression (28) we obtain equality:

$$
0=-\frac{H \rho(y)}{y^{2}}-\frac{|u| \rho^{\prime}(y)}{\rho(y)}
$$

which can only be true if $\rho^{\prime}(y)$ is negative.

4. The two expressions are easily obtained as follows:

- solve the equation $0=-\frac{H \rho\left(y_{s}\right)}{y_{s}^{2}}-\frac{|u| \rho^{\prime}\left(y_{s}\right)}{\rho\left(y_{s}\right)}$ for $H$ and set $u=K$ since $\dot{y}=0$ along a singular arc;

- the relation $H=q_{0}(u(0)-K)=q_{0}(1-K)$ yields the expression of $q_{0}$;

- finally, writing $H$ along a singular arc, $H=p_{0} y_{s}-\frac{y_{s}}{\rho\left(y_{s}\right)} K$, provides the expression of $p_{0}$.

Based on the informations gathered in Lemma 7, small time optimal trajectories are searched following the methodology exposed in Section 4.2.2. This is illustrated in Figure 14.

Finally, we propose the following methodology in order to address the optimal control problem $\left(\mathcal{P}_{1}\right)$ in the case of the road profile displayed in Figure 12.

1. Consider strategy (26). The sign of $\frac{\partial \rho}{\partial y}\left(y\left(t_{c}\right)\right)$ determines which strategy between (26) and (29) is relevant. We denote by $\bar{T}_{f}$ the corresponding final time.

2. For $T_{f}>\bar{T}_{f}$, either strategies of the form (27) or strategies of the form (30) are extremal and therefore candidate to optimality. Items (1) and (2) are illustrated in Figure 13.

3. For $T_{\min }<T_{f}<T_{b a c k}$, compute a forward extremal trajectory as in Section 4.2.2. As explained in Lemma 7, such trajectories are optimal extremals. This is illustrated in Figure 14 with a small time trajectory having no singular arc.

4. For $T_{\min }<T_{f}<\bar{T}_{f}$, as in the preceding item, forward extremal trajectory as in Section 4.2.2 can be searched. This is illustrated in Figure 15 with an extremal trajectory having a two-step braking behaviour.

5. Finally, with the help of a numerical study, extremal trajectories having backward arcs are searched for. For instance, for $T_{f}=4.48$, we didn't find any extremal having a backward arc and as such, the trajectory displayed in Figure 15 is considered optimal. Extremal trajectories having backward arcs are shown in Figures 16 and 17.

In order to better understand this last step, numerical exploration was performed via the manipulation of the values of $p_{0}$ and $q_{0}$. As expected, extremal trajectories having backward arcs do exist. An interesting fact being that such extremals are not necessarily optimal. In Figure 16, an example of an optimal trajectory with backward arcs is displayed. The yield function is the same as the one used to determine the trajectory of Figure 13. In this case, the backward strategy of Figure 16 costs less than the forward strategy of the form (30) - since the arcs with $y=0$ have a null running cost, strategy (30) costs the same regardless of the value of $T_{f}>T_{f}^{1, s, 0}$.

On the other hand, Figure 17 shows an extremal trajectory having backward arcs obtained for a yield function such that $\rho(0)$ is 20 times larger than the one used in Figure 16 -this is 
the only parameter that is changed in the design of $\rho$. The maximums of the two cost functions are the same, and are reached for the same values of $y$. In this case, the cost associated to the strategy (30) is equal to 0.73 , and the strategy of Figure 17 is not optimal.

Figures $16 \mathrm{~d}$ and $17 \mathrm{~d}$ highlight the values of the yield function that are visited when $u=$ -1 . In Figure 16d, the blue, black and green parts correspond to the moments when $u=-1$ that are drawn using the same colour in Figure 16c. In Figure 17d, the blue and black parts correspond to the moments when $u=-1$ that are drawn using the same colour in Figure $17 \mathrm{c}$. Figures $16 \mathrm{e}$ and $17 \mathrm{e}$ highlight the values of the yield function that are visited when $u=1$. Similarly, a colour code is used to point out moments when $u=1$.

\section{Conclusion}

This energy consumption optimisation problem allowed us to study an optimal control problem with a running cost that includes an absolute value function and a yield function. This problem has been addressed in fixed time and decomposed into several case studies, three of which were considered in the present article. For each one of them, the influence of the yield function upon optimal trajectories have been discussed. In particular, in the case of a flat road and a null drag coefficient, the yield function plays no role. Then, when the drag coefficient isn't null anymore, singular arcs may appear even in the case of a constant yield function. We also noticed that for some yield functions two-step braking strategies are optimal. In the last case study, involving a non-flat road profile, the yield function plays a role since the occurrence of singular arcs heavily depends on the sign of the function's derivative.

Finally, subsequent works on this topic should include investigations on extremal trajectories having both backward and singular arcs, especially arcs such that $y$ is not constant. The introduction of the drag coefficient in the case of a non-flat road, and different road profiles should also be covered.

\section{A Proof of Lemma 1}

First of all, let us remark that both $\mathbb{R}^{n} \times L_{[0, T]}^{1}$ and $C_{[0, T]}^{0}$ are metrisable sets, and as such sequential continuity implies uniform continuity.

1. Let $\left(u_{n}(t)\right)_{n \in \mathbb{N}}$ be a sequence of controls that converges to $u(t)$ in $L_{[0, T]}^{1}$, and $x_{0}^{(n)}$ be a $\mathbb{R}^{n}$ sequence converging to $x_{0}$. We denote by $x_{n}(t)$ the trajectory of $\left(\Sigma_{0}\right)$ given by $x_{0}^{(n)}$ and $u_{n}(t)$, and $x(t)$ the trajectory given by $x_{0}$ and $u(t)$. In other words, we have:

$x_{n}(t)=x_{0}^{(n)}+\int_{0}^{t} F\left(x_{n}(\tau)\right)+u_{n}(t) G\left(x_{n}(\tau)\right) d \tau$, and $x(t)=x_{0}+\int_{0}^{t} F(x(\tau))+u(t) G(x(\tau)) d \tau$.

Let us now write down the expression of $E_{n}(t)=x(t)-x_{n}(t)$ :

$$
E_{n}(t)=\underbrace{\left(x_{0}-x_{0}^{(n)}\right)}_{A}+\underbrace{\int_{0}^{t} F(x)-F\left(x_{n}\right) d \tau}_{B}+\underbrace{\int_{0}^{t} u G(x)-u_{n} G\left(x_{n}\right) d \tau}_{C} .
$$

Expression $C$ is rewritten:

$$
C=\underbrace{\int_{0}^{t}\left(u-u_{n}\right) G(x) d \tau}_{C_{1}}+\underbrace{\int_{0}^{t} u_{n}\left(G(x)-G\left(x_{n}\right)\right) d \tau}_{C_{2}}
$$


Next

$$
\left\|E_{n}(t)\right\| \leq\|A\|+\|B\|+\left\|C_{1}\right\|+\left\|C_{2}\right\|
$$

When restricted to a compact subset, $X$ et $Y$ are both Lipschitz with constants $K_{1}$ and $K_{2}$ respectively, and we have the inequalities:

(a) for all $\bar{\varepsilon}>0$, there exists $\bar{N} \in \mathbb{N}$ such that $\forall n>\bar{N},\|A\|<\bar{\varepsilon}$;

(b) since $X$ is Lipschitz: $\|B\| \leq \int_{0}^{t}\left\|F(x)-F\left(x_{n}\right)\right\| d \tau \leq K_{1} \int_{0}^{t}\left\|E_{n}(\tau)\right\| d \tau$;

(c) on $[0, T], Y$ is bounded from above (by some constant $\tilde{K}$ ), which yields:

$$
\left\|C_{1}\right\| \leq \tilde{K} \int_{0}^{t}\left\|u_{n}(\tau)-u(\tau)\right\| d \tau
$$

(d) finally, for all $n,\left|u_{n}\right| \leq 1$, and since $Y$ is Lipschitz, we have $\left\|C_{2}\right\| \leq K_{2} \int_{0}^{t}\left\|E_{n}(\tau)\right\| d \tau$.

Therefore, inequality (31) becomes:

$$
\left\|E_{n}(t)\right\| \leq\left\|x_{0}-x_{0}^{(n)}\right\|+K_{1} \int_{0}^{t}\left\|E_{n}(\tau)\right\| d \tau+\tilde{K} \int_{0}^{t}\left\|u_{n}(\tau)-u(\tau)\right\| d \tau+K_{2} \int_{0}^{t}\left\|E_{n}(\tau)\right\| d \tau
$$

Since $\left(u_{n}\right)$ converges in $L_{[0, T]}^{1}$ : for all $\tilde{\varepsilon}>0$, there is a $\tilde{N}$ such that $\forall n>\tilde{N}: \tilde{K} \int_{0}^{t}\left\|u_{n}-u\right\| d \tau<$ $\tilde{\varepsilon}$. Thus, for $n>\max (\bar{N}, \tilde{N})$, inequality (32) is rewritten:

$$
\left\|E_{n}(t)\right\| \leq(\bar{\varepsilon}+\tilde{\varepsilon})+\left(K_{1}+K_{2}\right) \int_{0}^{t}\left\|E_{n}(\tau)\right\| d \tau \leq(\bar{\varepsilon}+\tilde{\varepsilon})+\left(K_{1}+K_{2}\right) \int_{0}^{T}\left\|E_{n}(\tau)\right\| d \tau .
$$

Gronwall's lemma, for $t \in[0, T]$ gives:

$$
\left\|E_{n}(t)\right\| \leq(\bar{\varepsilon}+\tilde{\varepsilon}) e^{\left(K_{1}+K_{2}\right) T} \quad \forall n>\tilde{N} .
$$

In other words, for a given $\varepsilon>0$, we can choose $(\bar{\varepsilon}+\tilde{\varepsilon})$ such that, for all $n>N=$ $\max (\bar{N}, \tilde{N}),\left\|E_{n}(t)\right\|<\varepsilon$ for all $t \in[0, T]$.

Which proves the continuity of $\Phi(.,$.$) .$

2. In this second part, we use the preceding continuity result and the continuity of $u \mapsto|u|$ for the $L_{[0, T]}^{1}$ topology.

As before, $u_{n}(t)$ is a $L_{[0, T]}^{1}$-sequence of controls converging to $u(t), x_{0}^{(n)}$ converges to $x_{0}$, and $x_{n}$ (resp. $x$ ) is the trajectory generated by $x_{0}^{(n)}$ and $u_{n}$ (resp. $x_{0}$ et $u$ ). Let us now write the difference:

$$
\begin{aligned}
\left|J\left(u_{n}\right)-J(u)\right| & =\left|\int_{0}^{T}\right| \psi\left(x_{n}\right) u_{n}\left|d t-\int_{0}^{T}\right| \psi(x) u|d t| \\
& =\left|\int_{0}^{T}\right| \psi\left(x_{n}\right)\left|\left(\left|u_{n}\right|-|u|\right) d t-\int_{0}^{T}\right| u\left|\left(\left|\psi\left(x_{n}\right)\right|-|\psi(x)|\right) d t\right|
\end{aligned}
$$

Since $x_{n}(t)$ tends uniformly to $x(t)$ in $C_{[0, T]}^{0}$, there are a constant $M>0$, and an integer $N(M)$ such that for all $n>N(M)$, we have $\left|\psi\left(x_{n}\right)\right|<M$. For a given $\varepsilon>0$, there are a $\operatorname{rank} N\left(\frac{\varepsilon}{2 M T}\right)$ and a rank $N\left(\frac{\varepsilon}{2 T}\right)$ such that; 
(a) ||$u_{n}|-| u||<\frac{\varepsilon}{2 M T}$ for all $n>N\left(\frac{\varepsilon}{2 M T}\right)$,

(b) ||$\psi\left(x_{n}\right)|-| \psi(x)||<\frac{\varepsilon}{2 T}$ for all $n>N\left(\frac{\varepsilon}{2 T}\right)$.

Consequently, there exists a rank $N(\varepsilon)$ such that for all $n>N(\varepsilon)$

$$
\left|J\left(u_{n}\right)-J(u)\right|<M \int_{0}^{T} \frac{\varepsilon}{2 M T} d t+\int_{0}^{T} \frac{\varepsilon}{2 T} d t=\varepsilon
$$

which gives us the sequential continuity of $J($.$) .$

\section{References}

[1] A. Agrachev and Y. Sachkov. Control Theory from the Geometric Viewpoint, volume 87 of Encyclopedia of Mathematical Sciences. Springer, 2004.

[2] W. Alt and C. Schneider. Linear-quadratic control problems with $\mathrm{L}^{1}$-control cost. Optim. Control Appl. Meth., 36(4):512-534, 2015.

[3] D. J. Bell and D. H. Jacobson. Singular Optimal Control Problems. Academic Press: London, 1975.

[4] B. Berret, C. Darlot, F. Jean, T. Pozzo, C. Papaxanthis, and J. P. Gauthier. The inactivation principle: Mathematical solutions minimizing the absolute work and biological implications for the planning of arm movements. PLOS Computational Biology, 4(10), 2008.

[5] B. Berret, J.-P. Gauthier, and C. Papaxanthis. How humans control arm movements. In Proceedings of the Steklov Institute of Mathematics, volume 261, pages 44-58, 2008.

[6] B. Bonnard and M. Chyba. Singular Trajectories and their Role in Control Theory, volume 40 of Mathématiques \& Applications. Springer-Verlag Berlin Heidelberg New-York, 2003.

[7] U. Boscain and B. Piccoli. Optimal Syntheses for Control Systels on 2-D Manifolds, volume 43 of Mathématiques \& Applications. Springer-Verlag Berlin Heidelberg New-York, 2004.

[8] Z. Chen. $\mathrm{L}^{1}$-optimality conditions for circular restricted three-body problems. Celest. Mech. Dyn. Astr., doi:10.1007/s10569-016-9703-2, 2016.

[9] Z. Chen, J.-B. Caillau, and Y. Chitour. L $\mathrm{L}^{1}$-minimization for mechanical systems. SIAM Journal on Control and Optimization, 54(3):1245-1265, 2016.

[10] F. Clarke. Functional Analysis, Calculus of Variations and Optimal Control. Springer-Verlag London, 2013.

[11] G. Fraser-Andrews. Finding candidate singular optimal controls: a state of the art survey. Journal of Optimization Theory and Applications, 60(2):25-57, 1989.

[12] T. D. Gillespie. Fundamentals of vehicle dynamics. Premiere Series Books. Society of Automotive Engineers Inc., 1992. 
[13] K. A. Grasse. On the relation between small-time local controllability and normal selfreachability. Mathematics of Control, Signal and Systems, 1992.

[14] A. N. Kolmogorov and S. V. Fomin. Elements of the Theory of Functions and Functional Analysis, Vol. 2. Graylock Press, Rochester, New-York, 1961.

[15] E. B. Lee and L. Markus. Foundations of optimal control theory. The SIAM series in Applied Mathematics. John Wiley and Sons, New York-London-Sydney, 1967.

[16] H. Maurer and MR. De Pinho. Optimal control of epidemiological seir models with $\mathrm{L}^{1}$ objectives and control-state constraints. <hal- 01101291>, Submitted on 8 Jan 2015.

[17] L. S. Pontryagin, V. G. Boltyanskii, R. V. Gamkrelidze, and E. F. Mishchenko. The Mathematical Theory of Optimal Processes. John Wiley and Sons, New York-London-Sydney, 1962.

[18] I. M. Ross. Modern Astrodynamics, chapter Space Trajectory Optimization and $L^{1}$-Optimal Control Problems, pages 155-188. Elsevier, 2006.

[19] K. D. Sebesta. Optimal observers and optimal control : improving car efficiency with Kalman et Pontryagin. PhD thesis, Université de Bourgogne; Université du Luxembourg, 2010.

[20] G. Vossen and H. Maurer. On $\mathrm{L}^{1}$-minimization in optimal control and applications to robotics. Optim. Control Appl. Meth., 27:301-321, 2006. 


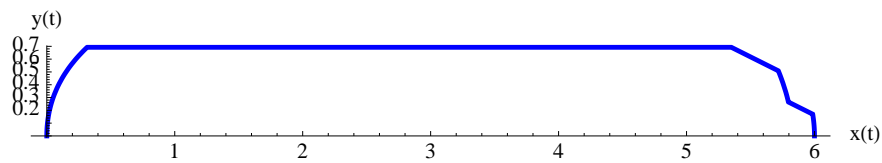

(a)

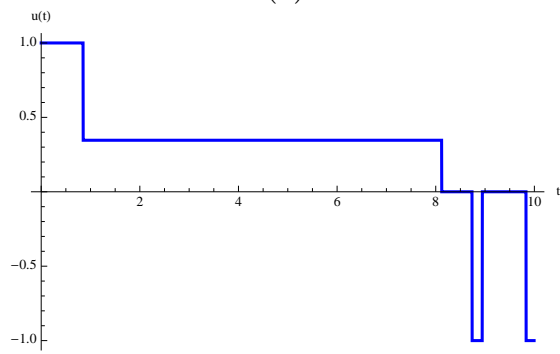

(b)

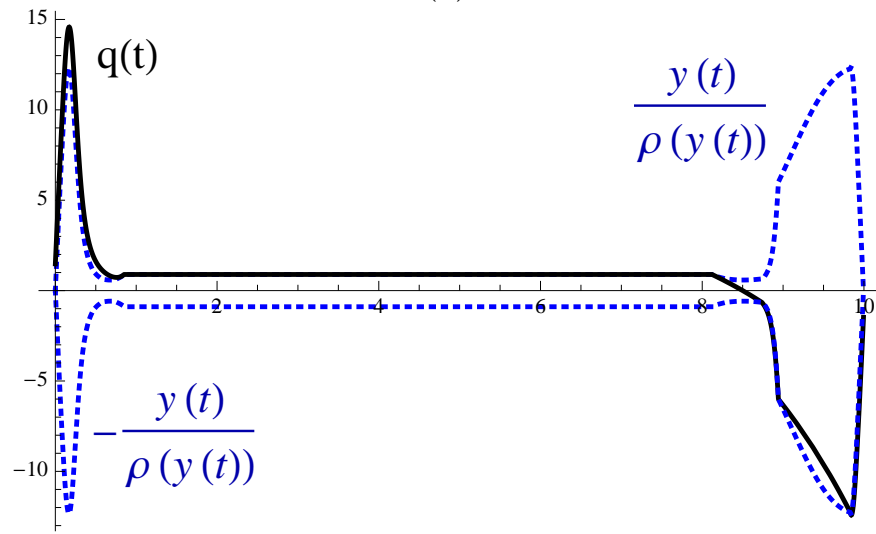

(c)

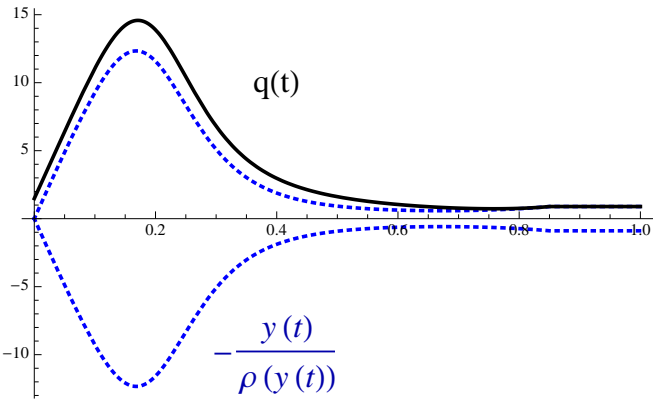

$(\mathrm{d})$

$\rho(\mathrm{y}(\mathrm{t}))$

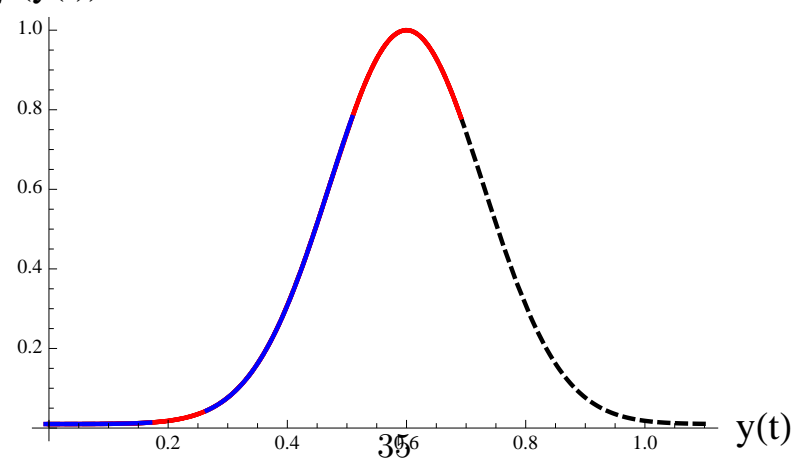

(f)

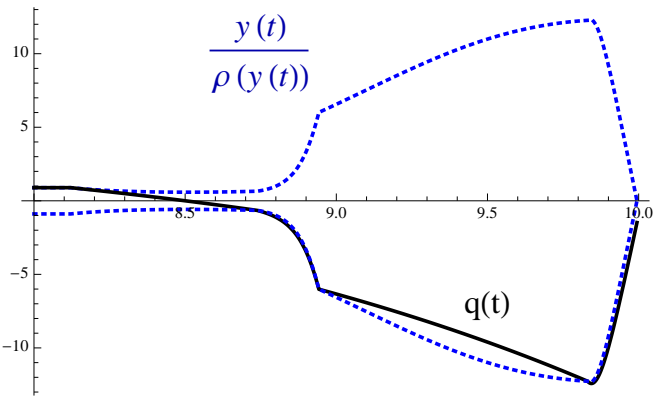

(e)

Fure 11: Optimal trajectory for the second case study with a non-constant yield function. Parameters: $\alpha=0.5, x_{f}=6, T_{\min } \approx 5.52, T_{\text {lim }} \approx 5.8$ and $T_{f}=10$. 


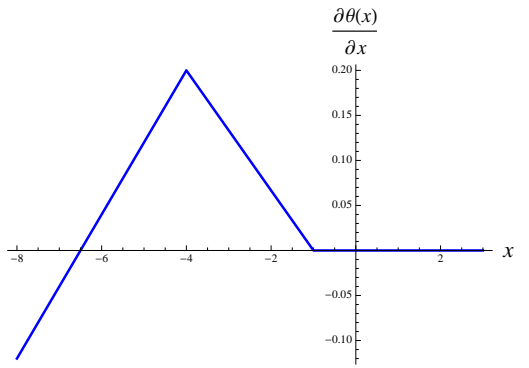

(a)

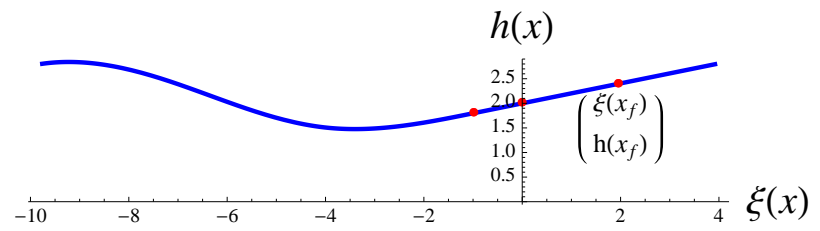

(b)

Figure 12: Derivative of $\theta$ with respect to $x$, and road profile in the $(\xi, h)^{t}$ coordinates. The red dots appearing on the road profile correspond to $x=-1, x=0$ and $x=x_{f}$ in this order. $T_{\text {min }} \approx 2.885, T_{f}^{1,0} \approx 5.01$.

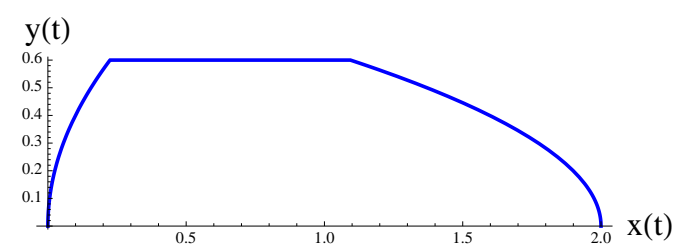

(a)

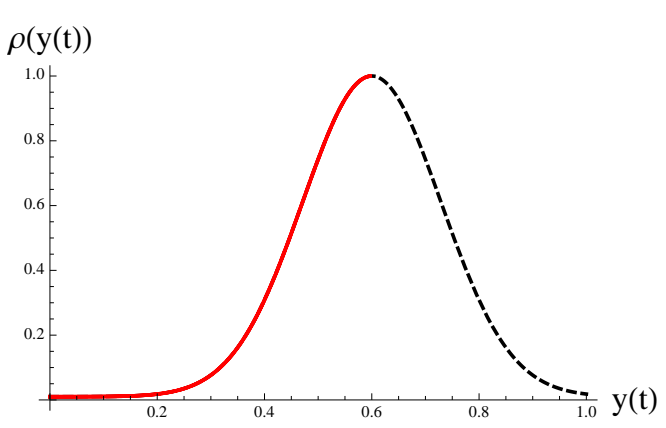

(c)

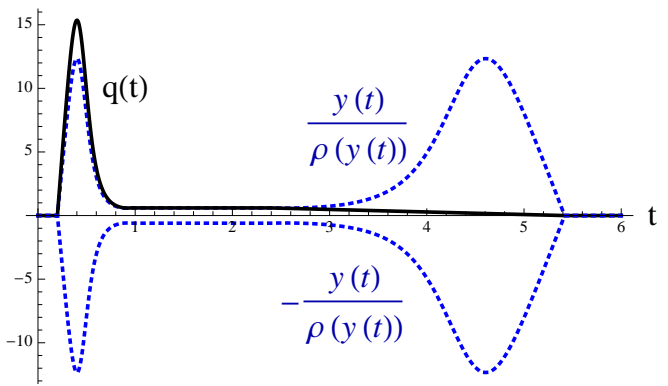

(b)

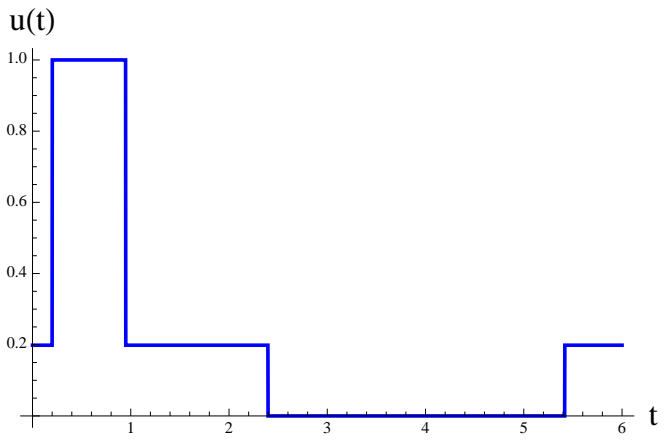

(d)

Figure 13: Optimal trajectory for the third case study. Parameters: $T_{f}^{1, s, 0} \approx 5.21$ and $T_{f}=6$. The associated cost equals 3.605 . 


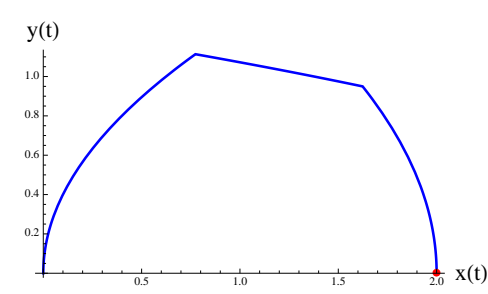

(a)

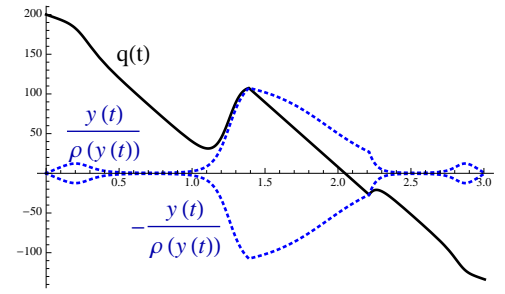

(b)

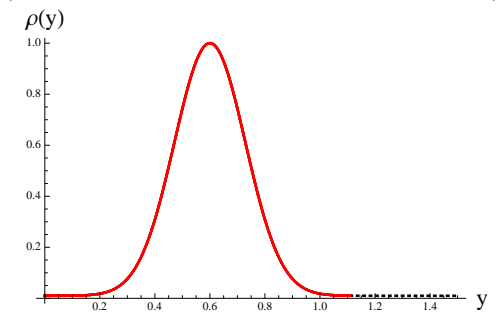

(c)

Figure 14: Optimal trajectory in the third case case study in small time. Parameters: $T_{\min } \approx 2.885, T_{f}=3$. 


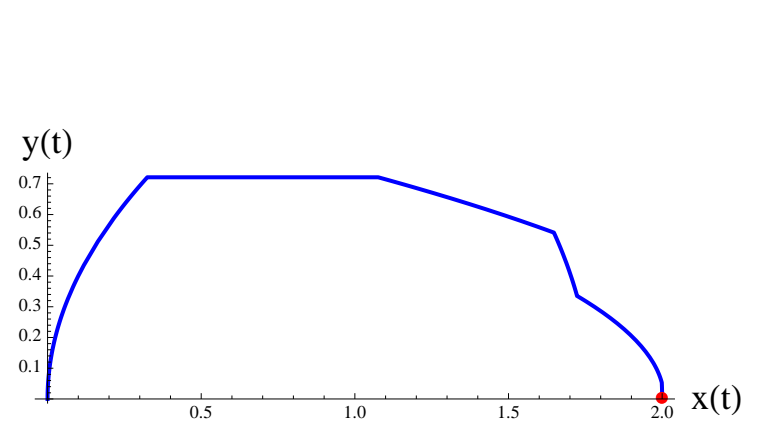

(a)

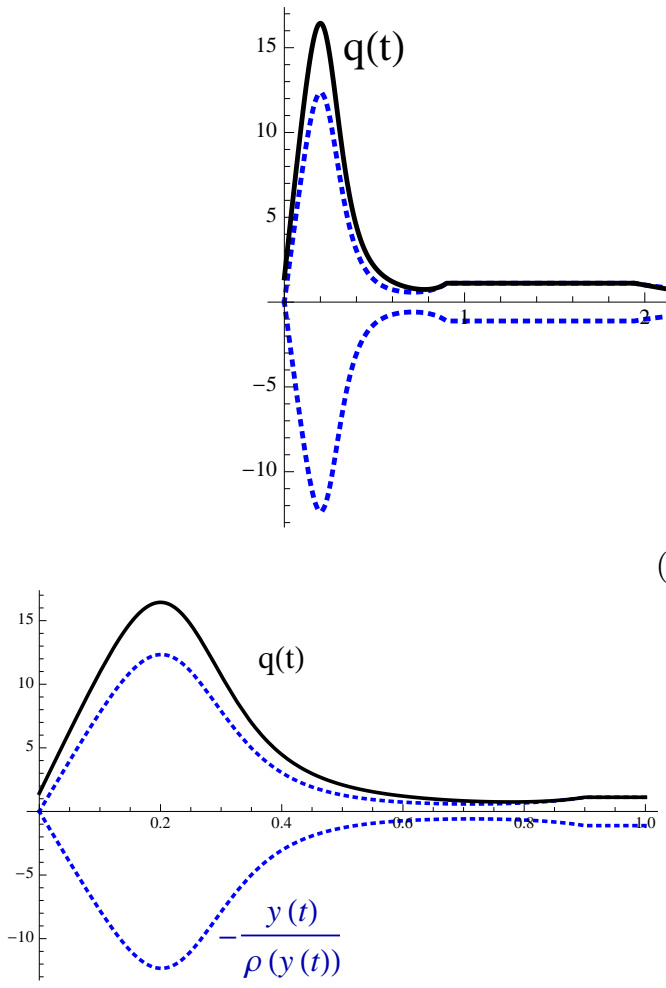

(c) (d)

$\rho(\mathrm{y}(\mathrm{t}))$

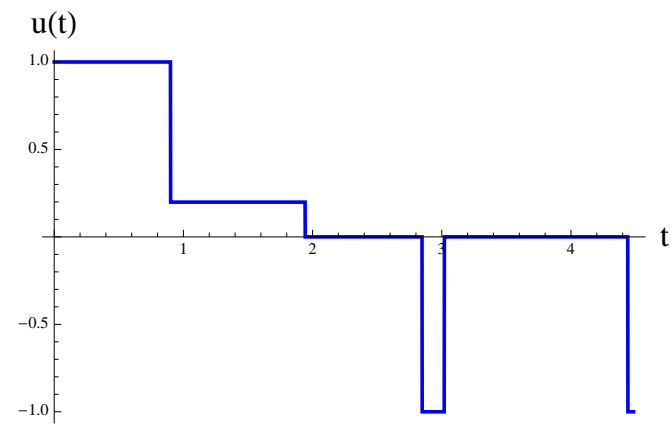

(b)

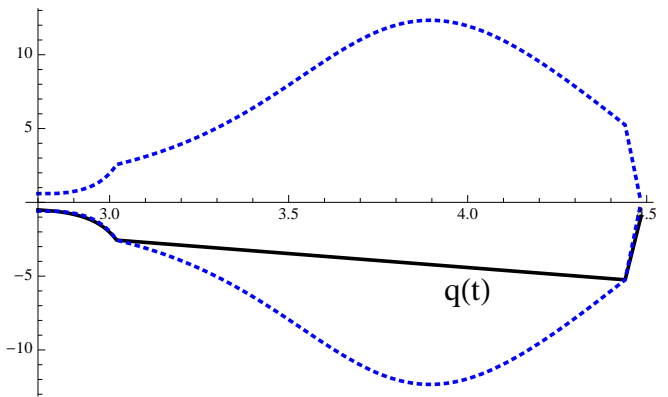

(e)

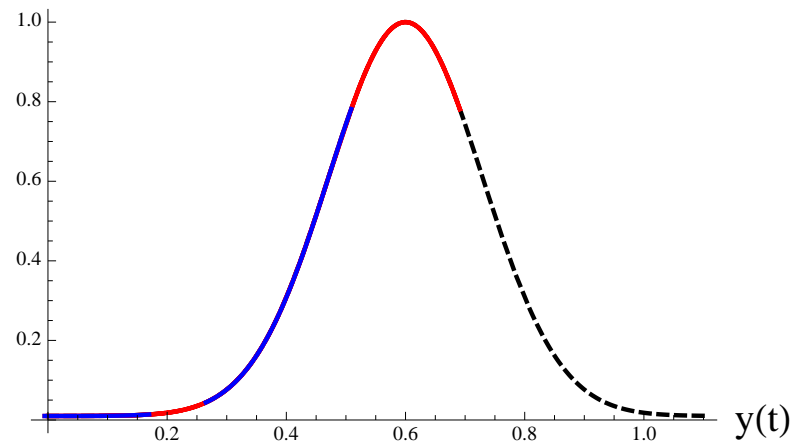

(f)

Figure 15: Optimal trajectory for the third case study for $T_{f}=4.48$. Figure 15f: the blue parts of the graph corresponds to the moments when $u=-1$. 


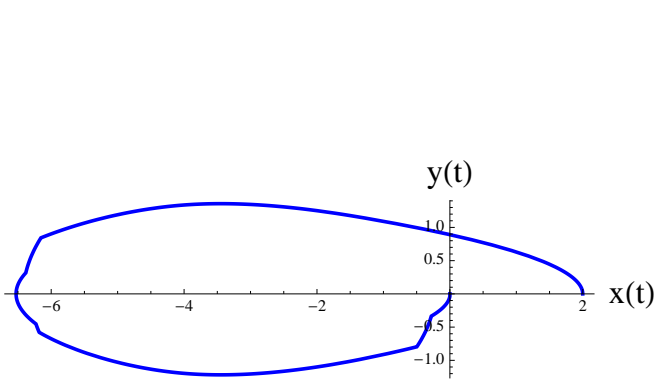

(a)

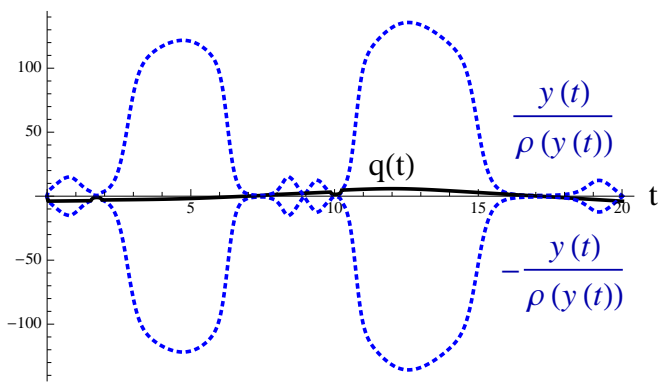

(b)

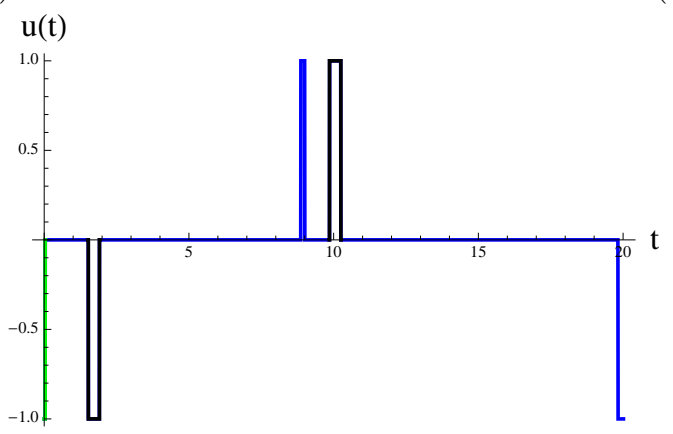

(c)

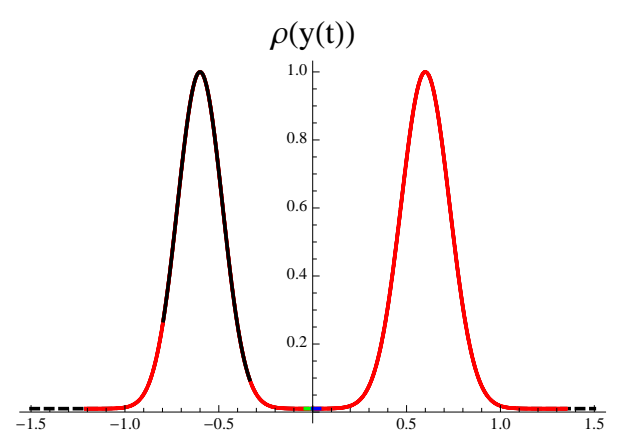

(d)

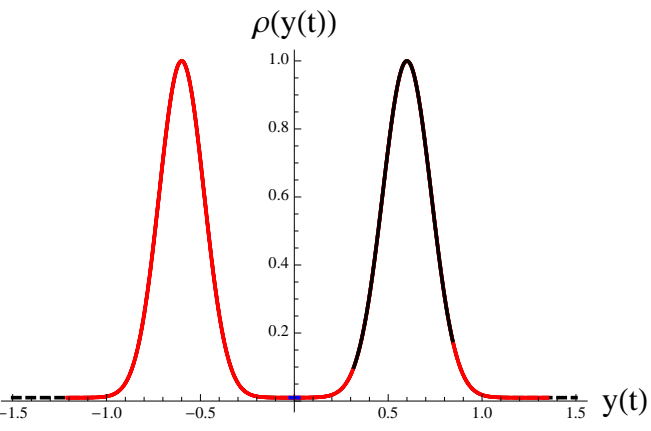

(e)

Figure 16: Optimal trajectory for the third case study for $T_{f} \approx 17.75$. The associated cost equals $\approx 1.61$ while it equals 3.605 for strategy $(30)$ — cf. Figure 13 . 


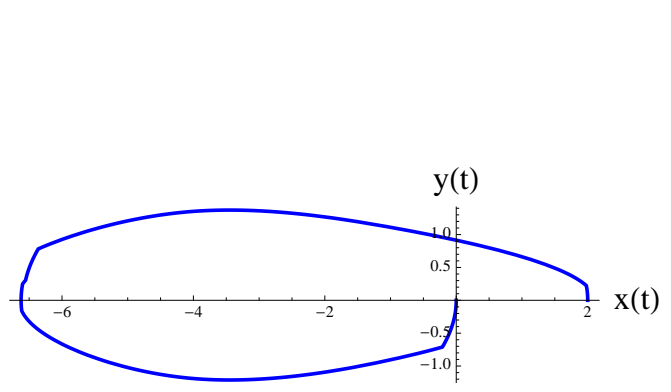

(a)

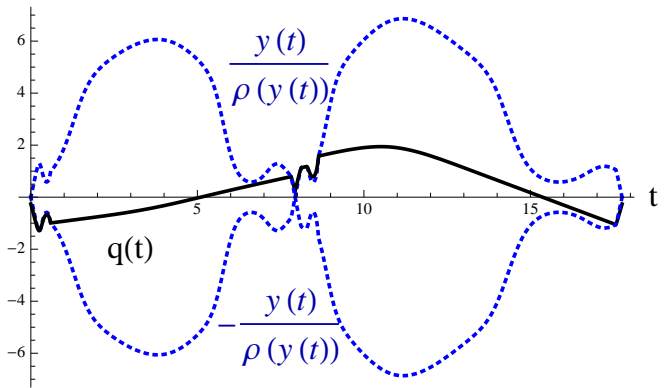

(b)

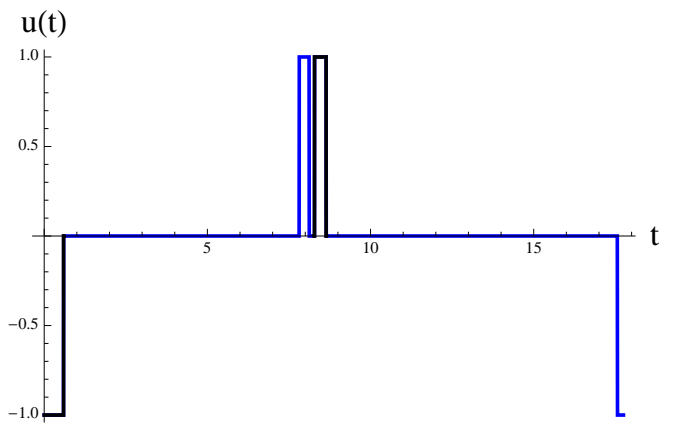

(c)

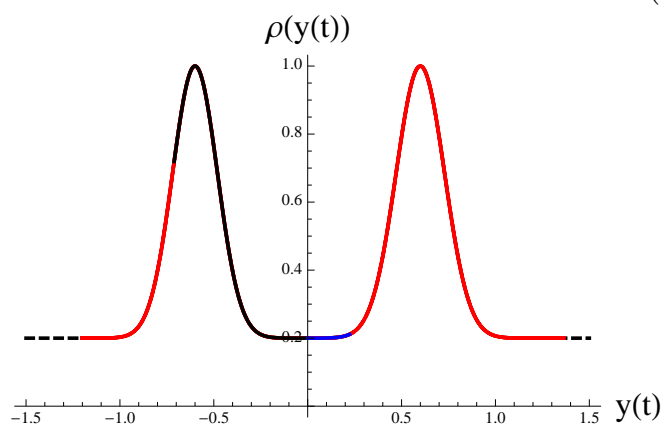

(d)

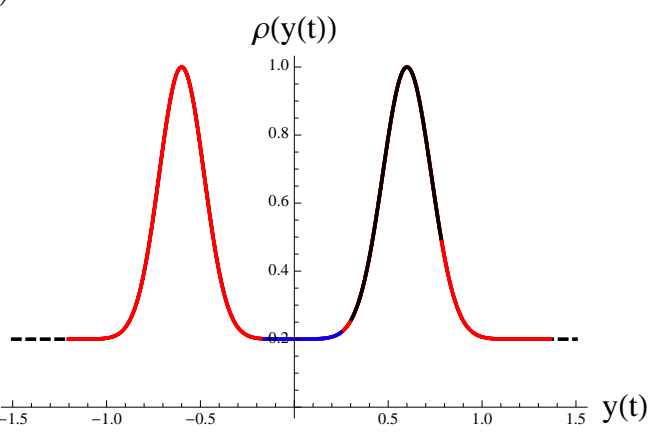

(e)

Figure 17: Extremal trajectory for the third case study for $T_{f} \approx 17.75$. The associated cost equals $\approx 1.02$ while it equals 0.73 for strategy (30) with the yield function of Figures $17 \mathrm{~d}$ and $17 \mathrm{e}$. 